\title{
Overview of The Ethnobotany on The Use of Plants as Potential Botanical Pesticides in Indonesia
}

\author{
Whisnu Febry Afrianto ${ }^{1 *}$, Rivandi Pranandita Putra ${ }^{2}$, Yasri Syarifatul Aini ${ }^{1}$ \\ ${ }^{1}$ Ecosystem and Biodiversity (Ecosbio), Jl. Merapi 02/01, Datengan, Grogol, Kediri, 64151 Indonesia; \\ ${ }^{2}$ Pre-Harvest Department, Indonesian Sugar Research Institute. Jl. Pahlawan No 25, Pasuruan, East \\ Java, 67126, Indonesia;
}

\author{
Article History \\ Received : December $17^{\text {th }}, 2021$ \\ Revised : December $29^{\text {th }}, 2021$ \\ Accepted : January $15^{\text {th }}, 2022$ \\ Published : January $22^{\text {th }}, 2022$ \\ *Corresponding Author: \\ Whisnu Febry Afrianto, \\ Ecosystem and Biodiversity \\ (Ecosbio), J1. Merapi 02/01, \\ Datengan, Grogol, Kediri, 64151 \\ Indonesia. \\ Email: \\ whisnu.afrianto@apps.ipb.ac.id
}

\begin{abstract}
Ethnobotany of Indonesian communities utilizes plants as botanical pesticides. Recently, there has been no comprehensive data and information related to the ethnobotany of plants as potential botanical pesticides on a nationwide scale. This paper aimed to depict an overview of ethnobotany as botanical pesticides in Indonesia. The comprehensive literature was collected from the 29 published articles and theses (doctoral, master's, and bachelor's degrees) in English and Bahasa Indonesia. After the data was validated, only 27 papers were related to the study topic. Most literature data is from the western part of Indonesia (Oriental Realm): Java $(n=10)$; followed by Kalimantan $(n=7)$, and Sumatra $(n=4)$. The results showed that 149 plant species were used as botanical pesticides. It consists of 130 species as an insecticide, 12 as a fungicide, 8 as a bactericide, 8 as a molluscicide, 3 as a rodenticide, and 2 as a nematicide. These species were dominated by the Compositae family (14 species) and plant parts used from leaf parts $(38.9 \%)$. However, 11 species have two to four functions: Archidendron pauciflorum, Areca catechu, Artocarpus heterophyllus, Caryota mitis, Cerbera manghas, Jatropha curcas, Melia azedarach, Morinda citrifolia, Pangium edule, Piper betle, and Piper ningrum. The data of ethnobotany can be used as database information for further research regarding the bioprospecting, formulation, efficacy, and conservation for sustainable use. Furthermore, the development of botanical pesticides is also an alternative to reduce synthetic/chemical pesticides to provide sustainable agriculture.
\end{abstract}

Keywords: botanical pesticides, ethnobotany, family plant, plant parts used, plant species

\section{Introduction}

Several negative impacts of chemical pesticides use have been reported, namely (1) impacts on health that are short-term effects (i.e., skin eye and skin irritation, dizziness, headaches, as well as nausea) to long-term effects (i.e., asthma, cancer, and diabetes) (Bassil et al. 2007; Ntzani et al. 2013; Sarwar 2015; Kim et al. 2017); (2) impacts on the environment that lead of the biodiversity loss (Geiger et al. 2010; Beketov 2013; Park et al. 2015; Mingo et al. 2016; Dudley et al. 2017; Brühl and Zaller 2019), as well as water, air, and soil pollution (Anderson et al. 2013; Belenguer et al. 2014; Riah et al. 2014; Meftaul et al. 2020); (3) impact on the economy that in the USA, an estimated $\$ 9.6$ billion of the economic damage was caused by the overuse of pesticides (Pimentel and Burgess 2014).
Based on the facts above, botanical pesticides can be an alternative to reduce synthetic/chemical pesticide use. The plant extracts contain insecticidal, antifungal, antibacterial, antivirus activity, antifeedant effects, insect growth regulation, repellence to pets, toxicity to nematodes, mites, and other pests (Seiber et al. 2014; Kumar and Singh 2015; Pavela and Benelli 2016). However, botanical pesticides still only reach a small number of pest control products globally. The shifting to botanical pesticides makes still slow progress because of the weakness of botanical pesticides, such as relatively quiet to kill, difficulty to kill the target pest directly, cost, not durable, challenges of production, less practical in application, lack of appropriate formulations, intolerant to sunlight now, and poor image based on previous low work of botanical pesticides (Glare et al. 2016). 
Many local people use wild species (plants, fungus, and animals) nearby because they can harvest freely, and it is available so diverse (Mardiastuti et al. 2021 a,b). The study on the dynamic of the knowledge of the connection between local people, biota, and their environmental resources is called Ethnobiology (Clement 1998; Iskandar 2017). One of the branches of ethnobiology is ethnobotany, which is defined as the usability of plants by the ethnic people for their daily needs (Prance 1991; Gaoue et al. 2017; Pandey and Tripathi 2017). Most of the studies of ethnobotany are still focus on the utilization of plants as foods (Sukenti et al. 2016; Cita 2020; Navia et al. 2020; Waroy and Utami 2020; Afrianto et al. 2021), medicines (Putri et al. 2016; Nahdi et al. 2016; Supiandi et al. 2019; Jadid et al. 2020), or rituals/myths (Iskandar et al. 2017; Erawan et al. 2018; Ratnani et al. 2021). It is still rare to study the use of ethnobotany for botanical pesticides purposess.

There was no comprehensive data and information related to ethnobotany as botanical pesticides nationwide. Some studies have investigated in small-scale sites or specific species/families. For example, Pratiwi and Nurlaeni (2021) investigate the potency of botanical pesticides of the Myrtaceae family collected in the Cibodas Botanical Garden. The research of botanical pesticides must be done to collect and explore how to receive national data (Mardiastuti et al. 2021 a). This paper aimed to depict an overview of ethnobotany as botanical pesticides in Indonesia. Integrating ethnobotany, bioprospecting, and conservation will bring sustainable use of plant resources in the future (Afrianto et al. 2017; Afrianto et al. 2020).

\section{Materials and Methods}

\section{Procedures}

The comprehensive literature was collected from the 29 published papers and theses (doctoral, master's, and bachelor's degrees) in English and Bahasa Indonesia. After the data was validated, there were only 27 works related to the study topic. Literature data were from the western part of Indonesia (Oriental Realm): Java $(n=10)$, followed by Kalimantan $(n=7)$, and
Sumatra $(n=4)$. A small number of literature data were collected from Nusa Tenggara $(n=4)$, Sulawesi $(n=3)$, and Papua $(n=1)$. Mainly, the paper published was in Indonesia Language.

\section{Data analysis}

The data was organized and analyzed based on plant species, local name, use category, the family of species, and part of plants used. The use category of botanical pesticides was grouped as insecticide, bactericide, rodenticide, nematicide, and molluscicide. Several data of plant parts used were not defined in the papers. Thus it was categorized as undefined.

\section{Results and Discussion}

\section{Plant diversity}

Based on the results of the identification and review conducted, in total, there were 149 species commonly used as botanical pesticides in Indonesia (Table 1). A total of 130 plant species used were dominated as insecticides, followed by fungicides (12), bactericides (8), molluscicides (8), rodenticides (3), and nematicides (2). These plants have potential as producers of botanical pesticides based on information on use, level of toxicity, and relevant literature information about the species concerned. On the other hand, 7 plant species were not identified until species level (taxonomically named as "sp."). This is because ethnobotany research relies only on interview results, especially in plant identification. Generally, the respondents only know the local name of the plant species. Furthermore, based on the literature review, three species had not recorded the local name.

Botanical pesticides have several characteristics: repellence, anti-feeding, deterrent activity, growth regulator, ovipositional deterrence, and feed deterrence (Hikal et al. 2017; Tripathi et al. 2009). Application of the botanical pesticides has to be an hourly application at the pre-harvest interval, and degradation only needs to take a few days (Hans and Saxena 2021). According to Isman and Grieneisen (2014), more than 20.000 papers were published regarding botanical insecticides from 1980 to 2012. 
Table 1. Diversity of plant used by the local people based on categories (black dots)

\begin{tabular}{|c|c|c|c|c|c|c|c|c|c|}
\hline Local Name & Scientific Name & Family & $\begin{array}{c}\text { Part of } \\
\text { Plant Used }\end{array}$ & 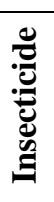 & 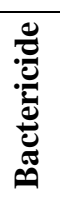 & 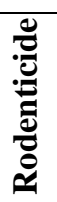 & 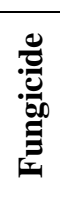 & 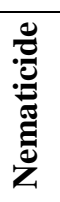 & 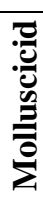 \\
\hline $\begin{array}{l}\text { Brotowali/Akar } \\
\text { Ali-ali }\end{array}$ & $\begin{array}{l}\text { Tinospora crispa (L.) } \\
\text { Hook. f. \& Thomson }\end{array}$ & Menispermaceae & Stem & $\circ$ & O & $\bullet$ & ○ & ० & O \\
\hline Cambai/sirih & Piper betle L. & Piperaceae & Leaf, stem & $\bullet$ & o & ० & $\bullet$ & ○ & ○ \\
\hline Jengkol & $\begin{array}{l}\text { Archidendron } \\
\text { pauciflorum (Benth.) } \\
\text { I.C. Nielsen }\end{array}$ & Leguminosae & Fruit & $\circ$ & ० & $\bullet$ & ○ & O & $\bullet$ \\
\hline Jeruk purut & Citrus hystrix DC. & Rutaceae & Leaf & $\bullet$ & O & o & ○ & ० & O \\
\hline Kapok & $\begin{array}{l}\text { Ceiba petandra (L.) } \\
\text { Gaertn. }\end{array}$ & Bombaceae & Leaf & $\bullet$ & O & ० & ○ & ० & O \\
\hline Kemiri & $\begin{array}{l}\text { Aleurites moluccanus } \\
\text { (L.) Willd }\end{array}$ & Euphorbiaceae & Fruit, bark & $\bullet$ & O & O & ○ & ० & O \\
\hline Medang keladi & Litsea crassinervia & Lauraceae & Bark & $\bullet$ & O & O & ○ & ० & o \\
\hline Nangka & $\begin{array}{l}\text { Artocarpus } \\
\text { heterophyllus Lam. }\end{array}$ & Moraceae & $\begin{array}{l}\text { Fruit, bark, } \\
\text { root }\end{array}$ & $\bullet$ & ० & 0 & $\circ$ & $\bullet$ & O \\
\hline Pinang & Areca catechu L. & Arecaceae & Leaf & $\bullet$ & O & ० & ○ & $\bullet$ & ० \\
\hline $\begin{array}{l}\text { Terong bulat } \\
\text { hijau }\end{array}$ & Solanum sp. & Solanaceae & Leaf. seed & $\bullet$ & 0 & o & ○ & ० & ० \\
\hline Jejer & Derris sp. & Leguminosae & Root & $\bullet$ & ० & o & ○ & ० & O \\
\hline Kabau & $\begin{array}{l}\text { Pithecellobium } \\
\text { bubalinum (Jack) } \\
\text { Benth. }\end{array}$ & Leguminosae & Peel & $\bullet$ & o & ○ & ○ & 0 & O \\
\hline Durian & Durio zibethinus L. & Malvaceae & Bark & $\bullet$ & ० & o & o & o & ० \\
\hline Puar kilat & Globba sp. & Zingiberaceae & Leaf & $\bullet$ & ० & ○ & ○ & $\circ$ & ० \\
\hline Sitawar & $\begin{array}{l}\text { Costus speciosus } \\
\text { (J.Koenig) Sm. }\end{array}$ & Zingiberaceae & Leaf & $\bullet$ & ० & $\circ$ & ○ & ० & ० \\
\hline Legundi & Vitex trifolia $\mathrm{L}$. & Lamiaceae & Leaf & $\bullet$ & ० & ○ & ○ & $\circ$ & ० \\
\hline Lengkonai & $\begin{array}{l}\text { Selaginella plana } \\
\text { (Desv. ex Poir.) } \\
\text { Hieron. }\end{array}$ & Selaginellacea & Leaf, stem & $\bullet$ & O & ○ & ० & ० & o \\
\hline Sirsak & Annona muricata L. & Annonaceae & $\begin{array}{l}\text { Leaf, seed, } \\
\text { root }\end{array}$ & $\bullet$ & ० & o & ○ & ० & o \\
\hline Jior/johar & $\begin{array}{l}\text { Senna siamea (Lam.) } \\
\text { H. S. Irwin \& } \\
\text { Barneby }\end{array}$ & Leguminosae & Bark & $\bullet$ & ० & ○ & ○ & ० & ० \\
\hline Ingul/ungil & $\begin{array}{l}\text { Toona sureni } \\
\text { (Blume) Merr. }\end{array}$ & Meliaceae & Leaf & $\bullet$ & ० & o & ○ & ० & o \\
\hline Gadung & $\begin{array}{l}\text { Dioscorea hispida } \\
\text { Dennst. }\end{array}$ & Dioscoreaceae & Tuber & $\bullet$ & ० & o & ○ & ० & O \\
\hline Uwi alas & Dioscorea alata $\mathrm{L}$. & Dioscoreaceae & Tuber & $\bullet$ & ० & ○ & ० & ० & ० \\
\hline $\begin{array}{l}\text { Lareng/tubak } \\
\text { raong }\end{array}$ & $\begin{array}{l}\text { Derris elliptica } \\
\text { (Wall.) Benth. }\end{array}$ & Leguminosae & Root & $\bullet$ & O & ० & ○ & ० & ० \\
\hline Tuwa & Croton tiglium $\mathrm{L}$. & Euphorbiaceae & Root & $\bullet$ & ० & ० & ○ & $\circ$ & $\circ$ \\
\hline Sarang semut & $\begin{array}{l}\text { Myrmecodia armata } \\
\text { DC. }\end{array}$ & Rubiaceae & Undefined & $\bullet$ & O & ○ & ○ & ० & ० \\
\hline Berkebo & Tersntroemia toquin & Theaceae & Undefined & $\bullet$ & ० & ○ & ○ & O & O \\
\hline Mengkudu & Morinda citrifolia L. & Rubiaceae & Leaf & $\bullet$ & o & $\circ$ & ○ & o & $\bullet$ \\
\hline
\end{tabular}




\begin{tabular}{|c|c|c|c|c|c|c|c|c|c|}
\hline Local Name & Scientific Name & Family & $\begin{array}{c}\text { Part of } \\
\text { Plant Used }\end{array}$ & 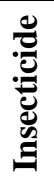 & 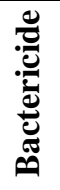 & 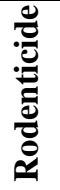 & 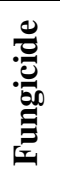 & 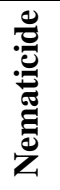 & 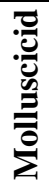 \\
\hline Tomat & $\begin{array}{l}\text { Solanum } \\
\text { lycopersicum L. }\end{array}$ & Solanaceae & Fuit & • & ० & ○ & $\circ$ & ० & ○ \\
\hline Ketepeng & $\begin{array}{l}\text { Senna alata }(\mathrm{L} .) \\
\text { Roxb. }\end{array}$ & Leguminosae & Leaf & • & ० & o & $\circ$ & ० & ○ \\
\hline Jarak & Jatropha curcas L. & Euphorbiaceae & Fruit & $\bullet$ & $\bullet$ & 0 & $\bullet$ & O & $\bullet$ \\
\hline Karet & $\begin{array}{l}\text { Hevea brasiliensis } \\
\text { (Willd. ex A.Juss.) } \\
\text { Müll.Arg. }\end{array}$ & Euphorbiaceae & Seed & $\bullet$ & ० & ○ & ० & ० & ○ \\
\hline Daun katum & $\begin{array}{l}\text { Nephelium mangayi } \\
\text { Hiern }\end{array}$ & Rubiaceae & Leaf & $\circ$ & $\bullet$ & ० & ० & ० & ○ \\
\hline Kase & $\begin{array}{l}\text { Pometia pinnata } \\
\text { J.R.Forst. \& G.Forst. }\end{array}$ & Sapindaceae & Bark & $\circ$ & ० & ० & ० & ० & $\bullet$ \\
\hline Tembakau & Nicotiana sp. & Solanaceae & Leaf & $\bullet$ & ० & $\circ$ & ० & $\circ$ & ० \\
\hline Kecubung & Datura metel L. & Solanaceae & Leaf & $\bullet$ & ० & $\circ$ & o & ० & $\circ$ \\
\hline Kalimonteng & $\begin{array}{l}\text { Homalomena cordata } \\
\text { Schott }\end{array}$ & Araceae & Leaf & $\bullet$ & ० & ० & ० & ० & o \\
\hline Kepayang & $\begin{array}{l}\text { Pangium edule } \\
\text { Reinw. }\end{array}$ & Achariaceae & Leaf, fruit & $\bullet$ & ० & ० & ० & ० & $\bullet$ \\
\hline Tuba goyeng & $\begin{array}{l}\text { Justicia gendarussa } \\
\text { Burm.f. }\end{array}$ & Acanthaceae & Leaf & $\bullet$ & ० & ० & ० & ○ & ० \\
\hline Salumang & $\begin{array}{l}\text { Asimina triloba }(\mathrm{L} .) \\
\text { Dunal }\end{array}$ & Annonaceae & Leaf & $\bullet$ & ० & $\circ$ & ० & ० & $\circ$ \\
\hline Risi/tukas & Caryota mitis Lour. & Arecaceae & Leaf & $\bullet$ & o & o & o & o & $\bullet$ \\
\hline Bintaro & Cerbera manghas L. & Apocynaceae & Fruit & $\bullet$ & ० & $\bullet$ & o & ० & ० \\
\hline Buah sokma & Crescentia cujete L. & Bignoniaceae & Leaf, fruit & $\bullet$ & ० & ○ & o & ○ & $\circ$ \\
\hline Pepaya & Carica papaya L. & Caricaceae & Leaf & $\bullet$ & ० & ० & o & ० & o \\
\hline Tegari & Dianella sp. & Liliaceae & Root & $\bullet$ & o & o & o & o & o \\
\hline Mimba & $\begin{array}{l}\text { Azadirachta indica } \\
\text { A.Juss. }\end{array}$ & Meliaceae & Leaf, seed & $\bullet$ & ० & $\circ$ & $\circ$ & $\circ$ & $\circ$ \\
\hline Serai & $\begin{array}{l}\text { Cymbopogon citratus } \\
\text { (DC.) Stapf }\end{array}$ & Poaceae & Leaf & $\bullet$ & ० & ० & ० & ० & ० \\
\hline Pohon redan & Nephelium maingayi & Rubiaceae & Bark & $\circ$ & ० & ○ & ० & ○ & $\bullet$ \\
\hline Jelatang & Urtica dioica $\mathrm{L}$. & Urticaceae & Leaf & $\circ$ & ० & o & o & ० & $\bullet$ \\
\hline Kecombrang & $\begin{array}{l}\text { Nicolaia speciosa } \\
\text { (Blume) Horan. }\end{array}$ & Zingiberaceae & Leaf & $\bullet$ & o & ० & ० & ० & ० \\
\hline Adas & $\begin{array}{l}\text { Foeniculum vulgare } \\
\text { Mill. }\end{array}$ & Apiaceae & $\begin{array}{l}\text { Stem, } \\
\text { flower, leaf }\end{array}$ & $\bullet$ & ○ & ० & ० & ० & ० \\
\hline Asam jawa & Tamarindus indica $\mathrm{L}$. & Leguminosae & Fruit & o & o & 0 & $\bullet$ & 0 & o \\
\hline Bandotan & $\begin{array}{l}\text { Ageratum conyzoides } \\
\text { (L.) L. }\end{array}$ & Compositae & Tuber & $\circ$ & $\circ$ & $\circ$ & $\bullet$ & $\circ$ & ० \\
\hline Bawang putih & Allium sativum L. & Amaryllidaceae & Tuber & $\circ$ & o & o & $\bullet$ & o & o \\
\hline Talas & $\begin{array}{l}\text { Colocasia esculenta } \\
\text { (L.) Schott }\end{array}$ & Araceae & $\begin{array}{l}\text { Leaf, stem, } \\
\text { root, fruit }\end{array}$ & $\bullet$ & ० & ○ & $\circ$ & ○ & ० \\
\hline $\begin{array}{l}\text { Belimbing } \\
\text { wuluh }\end{array}$ & Averrhoa bilimbi L. & Oxalidaceae & Leaf & $\circ$ & ० & $\circ$ & $\bullet$ & $\circ$ & $\circ$ \\
\hline Ceremei & $\begin{array}{l}\text { Phyllanthus acidus } \\
\text { (L.) Skeels }\end{array}$ & Phyllanthaceae & Fruit & $\bullet$ & ○ & ○ & o & ○ & ○ \\
\hline
\end{tabular}




\begin{tabular}{|c|c|c|c|c|c|c|c|c|c|}
\hline Local Name & Scientific Name & Family & $\begin{array}{c}\text { Part of } \\
\text { Plant Used }\end{array}$ & 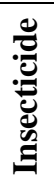 & 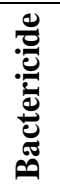 & 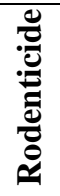 & 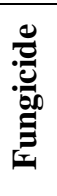 & 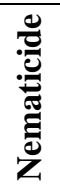 & 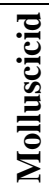 \\
\hline Dilem & $\begin{array}{l}\text { Pogostemon cablin } \\
\text { Benth. }\end{array}$ & Lamiaceae & $\begin{array}{l}\text { Root, stem, } \\
\text { leaf, flower }\end{array}$ & $\bullet$ & O & ○ & o & ○ & O \\
\hline Langsep & $\begin{array}{l}\text { Lansium domesticum } \\
\text { Corrêa }\end{array}$ & Meliaceae & Fruit & $\bullet$ & ○ & ○ & O & ○ & O \\
\hline Lidah buaya & $\begin{array}{l}\text { Aloe vera }(\mathrm{L} .) \\
\text { Burm.f. }\end{array}$ & Xanthorrhoeaceae & Stem & O & ○ & O & $\bullet$ & ○ & O \\
\hline Ganyong & $\begin{array}{l}\text { Canna edulis Ker } \\
\text { Gawl. }\end{array}$ & Cannaceae & $\begin{array}{l}\text { Leaf, } \\
\text { flower }\end{array}$ & $\bullet$ & O & o & 0 & ○ & ○ \\
\hline Jambu biji & Psidium guajava L. & Myrtaceae & Leaf & $\circ$ & ○ & ० & $\bullet$ & $\circ$ & ० \\
\hline Jambu monyet & $\begin{array}{l}\text { Anacardium } \\
\text { occidentale L. }\end{array}$ & Anacardiaceae & Flower & $\bullet$ & O & O & 0 & ○ & ○ \\
\hline Dringu & Acorus calamus L. & Acoraceae & $\begin{array}{l}\text { Root, stem, } \\
\text { leaf, tuber }\end{array}$ & $\bullet$ & ○ & ○ & O & ○ & O \\
\hline Kaliandra & $\begin{array}{l}\text { Calliandra } \\
\text { haematocephala } \\
\text { Hassk. }\end{array}$ & Leguminosae & Leaf & $\bullet$ & o & o & 0 & ○ & O \\
\hline Kamboja & $\begin{array}{l}\text { Plumeria acuminata } \\
\text { W.T.Aiton }\end{array}$ & Apocynaceae & Root & $\circ$ & $\bullet$ & ○ & $\circ$ & ○ & O \\
\hline Kunyit & Curcuma longa L. & Zingiberaceae & $\begin{array}{l}\text { Laef, } \\
\text { rhizome, } \\
\text { flower }\end{array}$ & $\bullet$ & o & O & o & $\circ$ & ○ \\
\hline Lada & Piper ningrum L. & Piperaceae & Fruit & $\bullet$ & $\bullet$ & $\circ$ & ० & $\circ$ & ० \\
\hline Lerak & Sapindus rarak DC. & Sapindaceae & Laef, fruit & $\circ$ & $\bullet$ & $\circ$ & o & $\circ$ & o \\
\hline Mindi & Melia azedarach L. & Meliaceae & Fruit, leaf & $\bullet$ & $\bullet$ & $\circ$ & $\bullet$ & $\circ$ & ० \\
\hline Patah tulang & Euphorbia tirucalli L. & Euphorbiaceae & Root, stem & $\bullet$ & ० & $\circ$ & ० & $\circ$ & ० \\
\hline Mojo & $\begin{array}{l}\text { Aegle marmelos (L.) } \\
\text { Corrêa }\end{array}$ & Rutaceae & Fruit, leaf & $\bullet$ & ० & $\circ$ & ० & ○ & ० \\
\hline Sambiloto & $\begin{array}{l}\text { Andrographis } \\
\text { paniculata (Burm.f.) } \\
\text { Nees }\end{array}$ & Acanthaceae & $\begin{array}{l}\text { Leaf, stem, } \\
\text { root }\end{array}$ & $\bullet$ & 0 & ○ & o & ○ & O \\
\hline Srikaya & Annona squamosa L. & Annonaceae & $\begin{array}{l}\text { Leaf, seed, } \\
\text { root }\end{array}$ & $\bullet$ & O & O & o & ○ & ○ \\
\hline Tapak liman & $\begin{array}{l}\text { Elephantopus scaber } \\
\text { L. }\end{array}$ & Compositae & Leaf, stem & $\bullet$ & ० & $\circ$ & ० & ○ & ० \\
\hline Tembelekan & Lantana camara L. & Verbenaceae & $\begin{array}{l}\text { Leaf, } \\
\text { flower }\end{array}$ & $\bullet$ & ० & ○ & ० & ○ & o \\
\hline Kemangi & Ocimum basilicum L. & Lamiaceae & Leaf & $\bullet$ & ० & $\circ$ & o & $\circ$ & ० \\
\hline Liak merah & $\begin{array}{l}\text { Zingiber officinale } \\
\text { Roscoe }\end{array}$ & Zingiberaceae & Undefined & $\bullet$ & ० & $\circ$ & ० & $\circ$ & ० \\
\hline $\begin{array}{l}\text { Bambu } \\
\text { tamiyang }\end{array}$ & $\begin{array}{l}\text { Schizostachyum } \\
\text { iraten Steud. }\end{array}$ & Poaceae & Leaf & $\bullet$ & ० & $\circ$ & ० & $\circ$ & 0 \\
\hline Andong merah & $\begin{array}{l}\text { Cordyline terminalis } \\
\text { (L.) Kunth }\end{array}$ & Asparagaceae & Leaf & $\bullet$ & o & ○ & ० & ○ & o \\
\hline Pinang hutan & Pinanga kuhlii Blume & Arecaceae & Leaf & $\bullet$ & 0 & ○ & 0 & $\circ$ & 0 \\
\hline Alang-alang & $\begin{array}{l}\text { Cymbopogon nardus } \\
\text { (L.) Rendle }\end{array}$ & Poaceae & Undefined & $\bullet$ & O & ○ & ० & ○ & O \\
\hline Bamban & $\begin{array}{l}\text { Donax canniformis } \\
\text { (G.Forst.) K.Schum. }\end{array}$ & Marantaceae & $\begin{array}{l}\text { Leaf, fruit, } \\
\text { stem }\end{array}$ & $\bullet$ & 0 & ○ & ० & ○ & O \\
\hline Teureup & $\begin{array}{l}\text { Artocarpus elasticus } \\
\text { Reinw. ex Blume }\end{array}$ & Moraceae & Undefined & $\bullet$ & 0 & ○ & O & ○ & ० \\
\hline
\end{tabular}




\begin{tabular}{|c|c|c|c|c|c|c|c|c|c|}
\hline Local Name & Scientific Name & Family & $\begin{array}{c}\text { Part of } \\
\text { Plant Used }\end{array}$ & 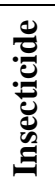 & 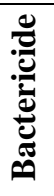 & 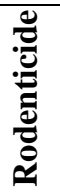 & 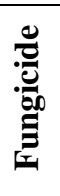 & 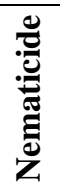 & 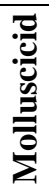 \\
\hline Aren & $\begin{array}{l}\text { Arenga } \\
\text { porphyrocarpa } \\
\text { (Blume ex Mart.) } \\
\text { H.E.Moore }\end{array}$ & Arecaceae & Undefined & $\bullet$ & ० & O & o & ○ & $\circ$ \\
\hline Marigold & Tagetes erecta L. & Compositae & Undefined & $\bullet$ & o & o & o & o & ० \\
\hline Daun bangle & $\begin{array}{l}\text { Zingiber cassumunar } \\
\text { Roxb. }\end{array}$ & Zingiberaceae & Rhizome & $\bullet$ & ० & ० & ० & 0 & $\circ$ \\
\hline Meniran & Phyllanthus niruri L. & Phyllanthaceae & Undefined & $\bullet$ & ० & ० & ० & ० & $\circ$ \\
\hline Paku rene & Selaginella sp. & Selaginellaceae & Leaf & $\bullet$ & $\circ$ & $\circ$ & ० & ० & $\circ$ \\
\hline Undefined & Cyperus pilosus Vahl & Cyperaceae & Undefined & $\bullet$ & 0 & 0 & 0 & 0 & 0 \\
\hline Keboro & $\begin{array}{l}\text { Halopegia blumei } \\
\text { (Körn.) K.Schum. }\end{array}$ & Marantaceae & Undefined & $\bullet$ & $\circ$ & ० & ० & 0 & ○ \\
\hline Kencur & $\begin{array}{l}\text { Kaempferia galanga } \\
\text { L. }\end{array}$ & Zingiberaceae & Undefined & $\bullet$ & ० & ० & ० & ० & $\circ$ \\
\hline Bawang kucai & $\begin{array}{l}\text { Allium } \\
\text { schoenoprasum } \mathrm{L} .\end{array}$ & Amaryllidaceae & Tuber & $\bullet$ & ० & ० & O & ० & $\circ$ \\
\hline Laos hutan & $\begin{array}{l}\text { Alpinia malaccensis } \\
\text { (Burm.f.) Roscoe }\end{array}$ & Zingiberaceae & Rhizome & $\bullet$ & ० & o & 0 & ० & $\circ$ \\
\hline Salam & $\begin{array}{l}\text { Syzygium polianthum } \\
\text { (Wight) Walp. }\end{array}$ & Myrtaceae & Undefined & $\bullet$ & ० & ० & 0 & ० & $\circ$ \\
\hline Jeruk purut & $\begin{array}{l}\text { Citrus maxima } \\
\text { (Burm.) Merr. }\end{array}$ & Rutaceae & Undefined & $\bullet$ & ० & ० & o & O & $\circ$ \\
\hline Lengkuas & $\begin{array}{l}\text { Alpinia galanga }(\mathrm{L} .) \\
\text { Willd. }\end{array}$ & Zingiberaceae & Fruit & $\bullet$ & $\circ$ & ० & O & ० & $\circ$ \\
\hline Senggani & $\begin{array}{l}\text { Melastoma candidum } \\
\text { D. Don }\end{array}$ & Melastomaceae & Undefined & $\bullet$ & ० & ० & ० & ○ & $\circ$ \\
\hline Miana & $\begin{array}{l}\text { Plectranthus } \\
\text { scutellarioides (L.) } \\
\text { R.Br. }\end{array}$ & Lamiaceae & Undefined & $\bullet$ & ० & ० & O & ० & $\circ$ \\
\hline Ketumbar jawa & Eryngium feotidum $\mathrm{L}$. & Apiaceae & Undefined & $\bullet$ & $\circ$ & $\circ$ & $\circ$ & ० & $\circ$ \\
\hline Pacing tawar & $\begin{array}{l}\text { Costus specious } \\
\text { (J.Koenig) Sm. }\end{array}$ & Costaceae & Undefined & $\bullet$ & ० & ० & ० & O & $\circ$ \\
\hline Hoya & $\begin{array}{l}\text { Hoya diversifolia } \\
\text { Blume }\end{array}$ & Apocynaceae & Leaf & $\bullet$ & ० & ० & O & ० & $\circ$ \\
\hline $\begin{array}{l}\text { Bambu awi } \\
\text { gede }\end{array}$ & $\begin{array}{l}\text { Gigantochloa } \\
\text { pseudoarundinacea } \\
\text { (Steud.) Widjaja }\end{array}$ & Poaceae & Shoot & $\bullet$ & ० & ० & 0 & O & $\circ$ \\
\hline Kelor & $\begin{array}{l}\text { Moringa oleifera } \\
\text { Lam. }\end{array}$ & Moringaceae & Root & $\bullet$ & ० & ○ & ० & O & $\circ$ \\
\hline Kelor hutan & $\begin{array}{l}\text { Boenninghausenia } \\
\text { albiflora (Hook.) } \\
\text { Rchb. ex Meisn. }\end{array}$ & Rutaceae & Leaf & $\bullet$ & ० & ० & O & O & $\circ$ \\
\hline Undefined & $\begin{array}{l}\text { Acanthospermum } \\
\text { hispidum DC. }\end{array}$ & Compositae & Undefined & $\circ$ & $\bullet$ & ० & O & 0 & $\circ$ \\
\hline Ajeran & Bidens pilosa L. & Compositae & Leaf & $\bullet$ & o & o & o & O & O \\
\hline Kirinyu & $\begin{array}{l}\text { Chromolaena } \\
\text { odorata (L.) } \\
\text { R.M.King \& H.Rob. }\end{array}$ & Compositae & Leaf & $\bullet$ & ० & o & O & 0 & $\circ$ \\
\hline
\end{tabular}




\begin{tabular}{|c|c|c|c|c|c|c|c|c|c|}
\hline Local Name & Scientific Name & Family & $\begin{array}{c}\text { Part of } \\
\text { Plant Used }\end{array}$ & 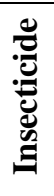 & 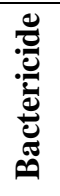 & 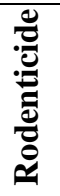 & 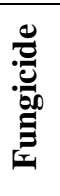 & 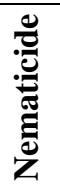 & 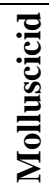 \\
\hline Babadotan & $\begin{array}{l}\text { Eleutheranthera } \\
\text { ruderalis (Sw.) } \\
\text { Sch.Bip. }\end{array}$ & Compositae & Undefined & $\bullet$ & ० & ० & $\circ$ & ० & ० \\
\hline Temu wiyang & $\begin{array}{l}\text { Emilia sonchifolia } \\
\text { (L.) DC. ex DC. }\end{array}$ & Compositae & Undefined & $\bullet$ & ० & ० & $\circ$ & ० & ० \\
\hline Undefined & $\begin{array}{l}\text { Grangea } \\
\text { maderaspatana }(\mathrm{L} .) \\
\text { Poir. }\end{array}$ & Compositae & Undefined & $\bullet$ & ० & ० & $\circ$ & ० & ० \\
\hline Undefined & $\begin{array}{l}\text { Sphaeranthus } \\
\text { africanus L. }\end{array}$ & Compositae & Undefined & $\bullet$ & ० & ० & $\circ$ & ० & ० \\
\hline Cagak langit & $\begin{array}{l}\text { Tridax procumbens } \\
\text { (L.) L. }\end{array}$ & Compositae & Undefined & $\bullet$ & ० & $\circ$ & ० & ० & O \\
\hline Sawi langit & $\begin{array}{l}\text { Vernonia cinerea }(\mathrm{L} .) \\
\text { Less. }\end{array}$ & Compositae & Leaf & $\circ$ & ○ & $\circ$ & $\bullet$ & ○ & ○ \\
\hline Seruni laut & $\begin{array}{l}\text { Wedelia biflora (L.) } \\
\text { DC. }\end{array}$ & Compositae & Leaf & $\circ$ & ○ & ○ & $\bullet$ & $\circ$ & ○ \\
\hline Pelawan & $\begin{array}{l}\text { Tristaniopsis } \\
\text { merguensis (Griff.) } \\
\text { Peter G.Wilson \& } \\
\text { J.T.Waterh. }\end{array}$ & Myrtaceae & Leaf, bark & $\bullet$ & o & 0 & $\circ$ & ० & O \\
\hline Pandan duri & $\begin{array}{l}\text { Pandanus tectorius } \\
\text { Parkinson ex Du Roi }\end{array}$ & Pandanaceae & Root, leaf & $\bullet$ & ○ & $\circ$ & $\circ$ & $\circ$ & ० \\
\hline Rumbia & $\begin{array}{l}\text { Metroxylon sago } \\
\text { Rottb. }\end{array}$ & Arecaceae & Leaf & $\bullet$ & ० & $\circ$ & $\circ$ & ० & ० \\
\hline Bungur & $\begin{array}{l}\text { Lagerstroemia } \\
\text { speciosa (L.) Pers. }\end{array}$ & Lythraceae & Leaf, stem & $\bullet$ & ० & $\circ$ & ० & $\circ$ & ० \\
\hline Simpur & Dillenia indica L. & Dilleniaceae & Leaf, bark & $\bullet$ & ० & $\circ$ & $\circ$ & $\circ$ & ० \\
\hline Salak hutan & $\begin{array}{l}\text { Eleiodoxa conferta } \\
\text { (Griff.) Burret }\end{array}$ & Arecaceae & Shoot & ○ & $\bullet$ & ० & ० & O & O \\
\hline Kelubuk & Ficus sp. & Moraceae & Leaf, fruit & $\circ$ & ० & ० & $\bullet$ & ० & ० \\
\hline Bijur laut & $\begin{array}{l}\text { Ipomoea pes-caprae } \\
\text { (L.) R. Br. }\end{array}$ & Convolvulaceae & $\begin{array}{l}\text { Leaf. stem, } \\
\text { root }\end{array}$ & $\bullet$ & ० & ० & $\circ$ & ० & O \\
\hline Mensirak & Ilex cymosa Blume & Aquifoliaceae & Root, leaf & $\bullet$ & ० & o & ० & ० & ० \\
\hline Api-api & $\begin{array}{l}\text { Avicennia marina } \\
\text { (Forssk.) Vierh. }\end{array}$ & Acanthaceae & Undefined & $\bullet$ & ० & ० & ० & $\circ$ & O \\
\hline Bakung & Crinum asiaticum L. & Amaryllidaceae & Undefined & $\bullet$ & o & ० & ० & ० & ० \\
\hline Tapak dara & $\begin{array}{l}\text { Catharanthus roseus } \\
\text { (L.) G. Don }\end{array}$ & Apocynaceae & Undefined & $\bullet$ & ० & o & ० & O & O \\
\hline Biduri & $\begin{array}{l}\text { Calotropis gigantea } \\
\text { (L.) Dryand. }\end{array}$ & Apocynaceae & Undefined & $\bullet$ & ० & ० & $\circ$ & ० & O \\
\hline Cemara & $\begin{array}{l}\text { Casuarina } \\
\text { equisetifolia } \mathrm{L} .\end{array}$ & Casuarinaceae & Undefined & $\bullet$ & o & ० & ० & ○ & O \\
\hline Nyamplung & $\begin{array}{l}\text { Calophyllum } \\
\text { inophyllum } \mathrm{L} .\end{array}$ & Clusiaceae & Undefined & $\bullet$ & ० & ० & ० & ० & O \\
\hline Ketepang & $\begin{array}{l}\text { Terminalia catappa } \\
\text { L. }\end{array}$ & Combretaceae & Undefined & $\bullet$ & ० & o & ० & O & ० \\
\hline Krasak & Ficus superba Miq. & Moraceae & Undefined & $\bullet$ & ० & ० & ० & ० & ० \\
\hline Cabe jawa & Piper caninum Blume & Piperaceae & Undefined & $\bullet$ & o & O & o & O & o \\
\hline Cabai & Capsicum annuum L. & Solanaceae & Fruit & $\bullet$ & o & 0 & o & O & O \\
\hline
\end{tabular}




\begin{tabular}{|c|c|c|c|c|c|c|c|c|c|}
\hline Local Name & Scientific Name & Family & $\begin{array}{c}\text { Part of } \\
\text { Plant Used }\end{array}$ & 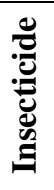 & 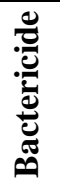 & 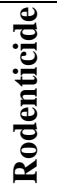 & 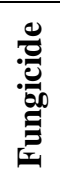 & 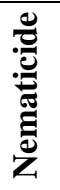 & 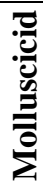 \\
\hline $\begin{array}{l}\text { Tembakau } \\
\text { rajangan }\end{array}$ & Nicotiana tabacum L. & Solanaceae & Leaf & $\bullet$ & ० & o & ○ & ○ & o \\
\hline Jagung & Zea mays $\mathrm{L}$. & Poaceae & Seed & $\bullet$ & $\circ$ & ० & ० & $\circ$ & ० \\
\hline Jeruk pahit & Citrus aurantium L. & Rutaceae & Leaf & $\bullet$ & 0 & 0 & o & 0 & 0 \\
\hline Sempuyung & $\begin{array}{l}\text { Hibiscus } \\
\text { heterophyllus Vent. }\end{array}$ & Malvaceae & Leaf & $\bullet$ & O & O & ○ & O & ० \\
\hline Krisan & $\begin{array}{l}\text { Chrysanthemum } \\
\text { indicum L. }\end{array}$ & Compositae & Leaf & $\bullet$ & ० & ० & ○ & ० & O \\
\hline Bandetan & $\begin{array}{l}\text { Clidemia hirta (L.) D. } \\
\text { Don }\end{array}$ & Melastomataceae & Leaf & $\bullet$ & o & ० & ○ & o & o \\
\hline $\begin{array}{l}\text { Cempedak } \\
\text { anyer }\end{array}$ & $\begin{array}{l}\text { Artocarpus dadah } \\
\text { Miq. }\end{array}$ & Moraceae & Leaf & $\bullet$ & o & ० & ○ & o & o \\
\hline Bayur & $\begin{array}{l}\text { Pterospermum } \\
\text { javanicum Jungh. }\end{array}$ & Malvaceae & Leaf & $\bullet$ & o & o & o & o & O \\
\hline Marak besi & $\begin{array}{l}\text { Macaranga } \\
\text { denticulata (Blume) } \\
\text { Müll.Arg. }\end{array}$ & Euphorbiaceae & Leaf & $\bullet$ & O & ○ & ○ & ० & O \\
\hline Tapak gajah & $\begin{array}{l}\text { Macaranga gigantea } \\
\text { (Rchb.f. \& Zoll.) } \\
\text { Müll.Arg. }\end{array}$ & Euphorbiaceae & Leaf & $\bullet$ & ० & ○ & $\circ$ & ० & ० \\
\hline $\begin{array}{l}\text { Marak Tiga } \\
\text { Jari }\end{array}$ & $\begin{array}{l}\text { Macaranga } \\
\text { hypoleuca (Rchb.f. \& } \\
\text { Zoll.) Müll.Arg. }\end{array}$ & Euphorbiaceae & Leaf & $\bullet$ & ० & ○ & ○ & ○ & ○ \\
\hline Gamal & $\begin{array}{l}\text { Gliricidia sepium } \\
\text { (Jacq.) Walp. }\end{array}$ & Leguminosae & Leaf & $\bullet$ & o & ० & ○ & ० & o \\
\hline Ngancar & $\begin{array}{l}\text { Planchonia valida } \\
\text { (Blume) Blume }\end{array}$ & Lechythidaceae & Stem & $\bullet$ & ० & o & ○ & ○ & 0 \\
\hline Ndarap & $\begin{array}{l}\text { Dysoxylum alliaceum } \\
\text { (Blume) Blume }\end{array}$ & Meliaceae & Stem & $\bullet$ & o & ○ & ○ & O & O \\
\hline Muku mada & $\begin{array}{l}\text { Musa acuminata } \\
\text { Colla }\end{array}$ & Musaceae & Leaf & $\bullet$ & $\circ$ & o & ○ & o & O \\
\hline Peca merah & Melia azedarach L. & Meliaceae & Leaf & $\bullet$ & $\circ$ & $\circ$ & o & o & o \\
\hline
\end{tabular}

\section{Family domination}

There were 11 families dominated where these families consisted of at least five plant species. These species were Apocynaceae (5 species), Arecaceae (6 species), Compositae (14 species), Euphorbiaceae (8 species), Leguminosae (9 species), Meliaceae (6 species), Moraceae (5 species), Poaceae (5 species), Rutaceae (5 species), Solanaceae (6 species), Zingiberaceae (9 species) (Figure 1). Dadang (1999) reported that plants with great potential for insect pest control are from Meliaceae, Rutaceae, Asteraceae, Annonaceae, Labiatae, Aristolochiaceae, Malvaceae, Zingiberaceae, and Solanaceae. This indicates that these plant species found can be developed as botanical pesticides in this study.

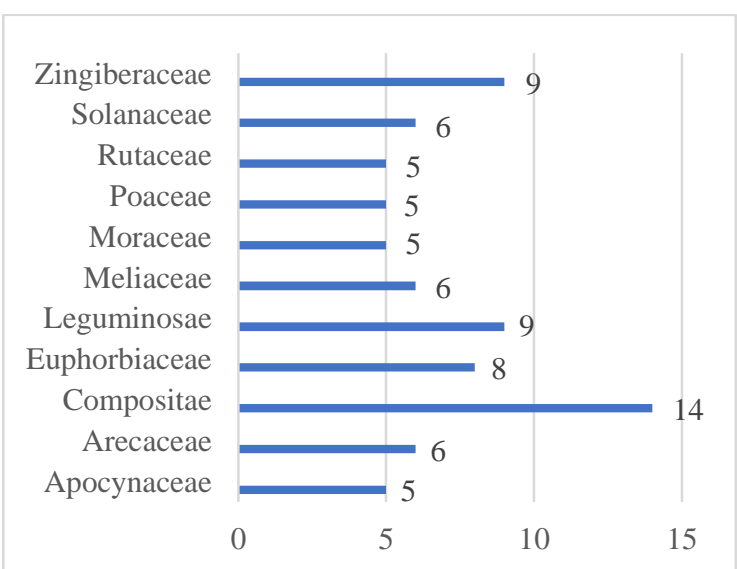

Figure 1. Plant family dominated as botanical pesticides in Indonesia 


\section{Plant parts used}

For this analysis, plant part data were only available for ten categories: flower, rhizome, seed, stem, leaf, bark, tuber, root, fruit, and unidentified. A high percentage of unidentified classes $(15.8 \%)$ was because many studies were not recorded part used in their studies. Available data showed that the majority of species used leaf (38.9\%), followed fruit (10.5\%), and root (8.9\%) (Figure 2). Several plants can use more than one part for botanical pesticides, which were 20 plant species with two parts, 8 plant species with three parts, and 3 plant species with four parts.

Over the past few years, plant extracts and essential oils have emerged as alternatives to synthetic insecticides for insect pest control. They result from plants that contain a bioactive chemical (Magierowicz et al. 2020). All different portions of plants can be used as essential oils. Beneficials of the essential oils have been reported for antifungal (Cavanagh 2007), antibacterial (O'Bryan et al. 2015), insecticide (Jankowska et al. 2018), nematicidal (Andrés et al. 2012), acaricidal (Peixoto et al. 2015). The application of essential oils is used as the fumigant and repellent (Tripati et al. 2009).

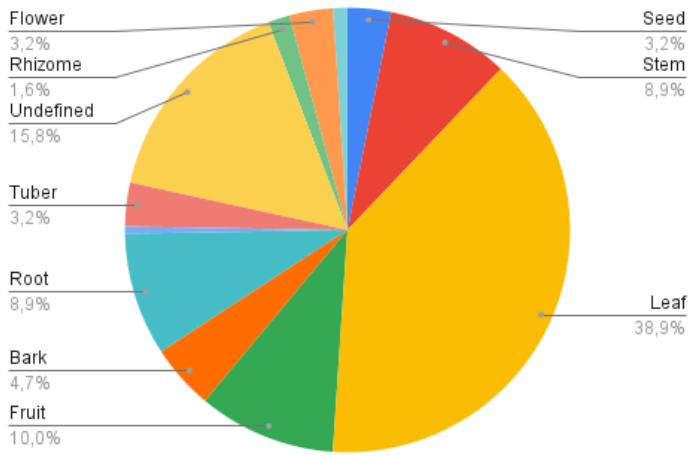

Figure 2. Plant parts used as botanical pesticides in Indonesia

\section{Multiple uses}

11 of 149 plant species had at least two to four functions: Archidendron pauciflorum, Areca catechu, Artocarpus heterophyllus, Caryota mitis, Cerbera manghas, Jatropha curcas, Melia azedarach, Morinda citrifolia, Pangium edule, Piper betle, and Piper ningrum (Tabel 2). Almost all plant species in this study were used for insecticide. Secondary metabolites that are essential oils of the plant can protect against herbivorous insect pests and pathogenic fungi (Apiaceae et al. 2011; Werrie et al. 2020).

Plant metabolites with diverse skeletons have been recorded to provide antimicrobial activities. Plant-derived that provide fungicidal and bactericidal compounds have relatively lower toxicity than insecticidal activity (Yoon et al. 2013). Moreover, plant-derived such as Cinnamaldehyde, 1-Glutamic acid + gammaaminobutyric acid, Jojoba oil, Laminarin, Milsana, Pink plume poppy powder extract, Essential oils, Fatty acids, Phenolic compounds, Alkaloids, and Glycosides can be used as antifungal activities. The botanical fungicides produce rapid biodegradation and high biocompatibility, lack of resistance, and structural diversity (Guo et al. 2021). For example, plant species, such as Piper betle, Jatropha curca, and Melia azederach, have been reported to have antifungal activity against Ascochyta rabiei, Aspergillus flavus, Aspergillus niger, Botrytis cinerea, Colletotrichum fragariae, Colletotrichum gloeosporioides, Corynospora cassicola, Fusarium oxysporum f. sp. Lycopersici, Fusarium oxysporum, f. sp. Melonis, Fusarium sambucinum, Fusarium solani var. coeruleum, Geotrichum spp., Pakospora pachyrhizi, Phytophthora sp., Rhizoctonia solani, Rigidoporous sp., Sclerotium spp., and Trichoderma spp. On the other hand, plant species Piper ningrum and Melia azederach have been reported to have antibacterial activity against Alternaria alternata, Bacillus nealsonii, $B$. subtilis, $B$. thuringiensis, Citrobacter freundiiEnterococcus faecalis, Erwinia cacticida, Escherichia coli, Microbacterium oleivorans, Plesiomonas shigelloides, Proteus mirabilis, Pseudomonas aeruginosa, $P$. syringae $p v$. syringae, Rathayibacter tritici, Shigella dysenteriae, S. flexneri, Staphylococcus aureus, and Xanthomonas campestris pv. campestris.

Not many studies have been conducted to examine the botanical pesticide for nematicide, molluciside, and rodenticide. Hurtada et al. (2012) conducted a study to examine the anthelmintic efficacy of Artocarpus heterophyllus on gastrointestinal nematodiasis on goats. Gastrointestinal nematodiasis cause a destructive impact on the productivity of goat farmings. This study has four genera of gastrointestinal nematodes to be tested, such as Trichostrongylus spp., Oesophagostomum spp., Haemonchus spp., and Bunostomum spp. The result shows that $A$. heterophyllus decoctions have high efficacy against nematode larvae at high concentrations.

One of the pests that result from a high risk of crop failure in rice plants is Mollusca. This pest eats the stems and leaves of 15-day-old rice. 
According to Sulistianingsih et al. (2014) shows the extract of Pangium edule seed at $100 \mathrm{ppm}$ can be used against various age levels. The concentration of $2.5 \%$ of seed $P$. edule extract with a hatching time of up to 28 days can inhibit hatching eggs of Pomacea canaliculata with average hatching of $63.84 \%$ (Noerfitryani 2017). The extract of $P$. edule fruit flesh has a more effect on mortality of Bradybaena similaris compared to the leaves and skin of the P. edule fruit (Noviyanti 2013). The peal of Archidendron pauciflorum with Dieffenbachia seguine leaf extract can be used against $P$. canaliculata at the optimum concentration of 15\% (Mawardi et al. 2018). The old seed extract of Jatropha curcas contains more toxicity to kill Mollusca than the young seed extract (Banjarnahor et al. 2016). Morinda citrifolia fruit at a concentration of $100 \mathrm{gr} / \mathrm{L}$ is the most significant concentration to kill mortality of $P$. caniculata (Chauhan et al. 2015). The extract of Areca catechu fruit at a concentration of $2.5 \%$ can kill young, preadult, and adult after 1, 4, and
5 days after application, respectively (Gassa 2011). The combination materials of $A$. indica and A. catechu are more effective because the mortality can reach $96.67 \%$ (Ma'wa and Hoesain 2020).

As pests, rats (Rattus argentiventer) can damage cultivated plants in a short time and cause considerable yield losses significant since the nursery, planting to the storage/warehouse, post-harvest. In the treatment, $600 \mathrm{~g}$ of $A$. pauciflorum seeds/liter of water and $800 \mathrm{~g}$ of $A$. pauciflorum seeds/liter of water can make Rattus argentiventer be abnormal growth where the effects are swelling of the stomach and neck (Pakki et al. 2009). Susanti et al. (2020) show that primary and secondary metabolites of Cerbera manghas can be used as a pest repellent.

Caryota mitis has not been proven as scientific as botanical pesticides. But, Community in Keranji uses $C$. mitis for insecticide and molluscicide. They take 10-20 C. mitis leaves to be dried and burned (Irtiawati et al. 2020).

Table 2. List of species that considered as multi-purpose species (i.e., has at least two out of six use categories) and scientific evidances

\begin{tabular}{|c|c|c|c|}
\hline $\begin{array}{l}\text { Scientific } \\
\text { Name }\end{array}$ & $\begin{array}{c}\text { Multiple } \\
\text { uses }\end{array}$ & Targets & Sources \\
\hline \multirow{2}{*}{$\begin{array}{l}\text { Archidendron } \\
\text { pauciflorum }\end{array}$} & 3 & Rodent: Rattus argentiventer & Pakki et al. 2009 \\
\hline & 6 & Mollusca: Pomecea canaliculata & Mawardi et al. 2018 \\
\hline \multirow[t]{2}{*}{ Piper betle } & 1 & $\begin{array}{l}\text { Insect: Aedes aegypti, Musca } \\
\text { domestica, Eudrilus eugeniae, } \\
\text { Callosobruchus maculatus, Sitophilus } \\
\text { zeamais, S. oryzae, Chrysomya } \\
\text { megacephala (larvae), C. bezziana } \\
\text { (larvae), Oxya serville }\end{array}$ & $\begin{array}{l}\text { Subaharan et al. 2021;Rahmawati 2020; Daswito } \\
\text { et al. 2019; Anisah and Sukesi 2018; Vasantha- } \\
\text { Srinivasan et al. 2018; Vasantha-Srinivasan et al. } \\
\text { 2017; Arambewel et al. 2010; Mohottalage et al. } \\
\text { 2007; Kumarasingha et al. 2002; Santhanam and } \\
\text { Nagarajan } 1990\end{array}$ \\
\hline & 4 & $\begin{array}{l}\text { Fungus: Colletotrichum sp., } \\
\text { Fusarium oxysporium sp., } \\
\text { Corynospora cassicola, Rigidoporous } \\
\text { sp. and Phytophthora sp., Rhizoctonia } \\
\text { sp., Fusarium solani var. coeruleum, } \\
\text { Colletotrichum fragariae }\end{array}$ & $\begin{array}{l}\text { Subrata et al. 2019; Achmad and Suryana 2015; } \\
\text { Wati et al. 2014; Subrata 2016; Ariyanti et al. } \\
\text { 2012; Arambewela et al. } 2010\end{array}$ \\
\hline \multirow[t]{2}{*}{$\begin{array}{l}\text { Artocarpus } \\
\text { heterophyllus }\end{array}$} & 1 & $\begin{array}{l}\text { Insect: Drosophila melanogaster, } \\
\text { Sitophilus oryzae, Spodoptera litura, } \\
\text { Scipophaga incertulas, Aedes aegypti, } \\
\text { Scirpophage incercutlas, and Culex } \\
\text { sp. }\end{array}$ & $\begin{array}{l}\text { Acero 2019; Firdaus and Kriswandana 2018; } \\
\text { Sharon et al. 2018; Nair and Kavrekar 2017; Gupta } \\
\text { et al. 2015; Upadhyay 2013; Shamim et al. } 2011\end{array}$ \\
\hline & 5 & $\begin{array}{l}\text { Nematode: Trichostrongylus spp., } \\
\text { Oesophagostomum spp., Haemonchus } \\
\text { spp. and Bunostomum spp. }\end{array}$ & Hurtada et al. 2014 \\
\hline $\begin{array}{l}\text { Caryota mitis } \\
\text { Lour. }\end{array}$ & $\begin{array}{l}1 \\
6\end{array}$ & $\begin{array}{l}\text { Insecticide: Undefinied } \\
\text { Mollusca: Undefined }\end{array}$ & \\
\hline $\begin{array}{l}\text { Cerbera } \\
\text { manghas } \mathrm{L} .\end{array}$ & 1 & $\begin{array}{l}\text { Insect: Anua indiscriminate, } \\
\text { Coptotermes Gestroi, Cryptotermes } \\
\text { Cynocephalus Sitophilus oryzae, }\end{array}$ & $\begin{array}{l}\text { Haryanta and Joeniarti 2021; Salbiah 2020; Tuhadi } \\
\text { et al. 2020; Susilo et al. 2019; Purwani et al. 2014; } \\
\text { Setiawan and Supriyadi 2014; Guswenrivo et al. } \\
\text { 2013; Tong et al. } 2007\end{array}$ \\
\hline
\end{tabular}


Afrianto, WF et al. (2022). Jurnal Biologi Tropis, 22 (1): 220 - 243

DOI: http://dx.doi.org/10.29303/jbt.v22i1.3266

\begin{tabular}{|c|c|c|}
\hline $\begin{array}{l}\text { Scientific } \\
\text { Name }\end{array}$ & $\begin{array}{l}\text { Multiple } \\
\text { uses }\end{array}$ & Targets \\
\hline & 3 & $\begin{array}{l}\text { Spodoptera litura. Helicoverpa } \\
\text { armigera } \\
\text { Rodent: Rattus argentiventer }\end{array}$ \\
\hline $\begin{array}{l}\text { Areca catechu } \\
\text { L. }\end{array}$ & 1 & $\begin{array}{l}\text { Insect: Callosbruchus analis, } \\
\text { Rhyzopertha dominica, Sitophilus } \\
\text { oryzae, Tribolium castaneum, } \\
\text { Crocidolomia pavonana, } \\
\text { Callosobruchus chinensis, } \\
\text { Chrysodeixis chalcites, Spodoptera } \\
\text { Liturra, Oryctes rhinoceros, } \\
\text { Helicoverpa Armigera, Bactrocera } \\
\text { carambolae, Nezara viridula, } \\
\text { Lasioderma Serricorne, Plutella } \\
\text { xylostellam, Nephotettix virescens, } \\
\text { Macrotermes gilvus } \\
\text { Mollusca: Pomacea canaliculata }\end{array}$ \\
\hline $\begin{array}{l}\text { Morinda } \\
\text { citrifolia L. }\end{array}$ & 1 & $\begin{array}{l}\text { Insecet: Spodoptera litura, Sitophilus } \\
\text { oryzae, Helicoverpa armigera, } \\
\text { Plutella xylostella, Sitophilus zeamais, } \\
\text { Crocidolomia binotalis, Crocidolomia } \\
\text { pavonana, Agrootis sp,, Anopheles } \\
\text { stephensi, Culex quinquefasciatus, } \\
\text { Aedes aegypti, Anopheles stephensi, } \\
\text { Blattella germanica, Tuta absoluta, } \\
\text { Callosobruchus maculatus, Cylas } \\
\text { formicarius, Drosophila melanogaster }\end{array}$ \\
\hline & 6 & Mollusca: Pomacea caniculata \\
\hline
\end{tabular}

Fadhillah 2020; Zailani 2015

Eka 2021; Subekti 2020; Kewa 2019; Lorenza

2019; Salbiah et al. 2019; Suswando 2019; Abbas et al. 2018; Mading et al. 2018; Rikardo and Nuryasin 2018; Dewi et al. 2017; Hayata 2017; Gobai, et al. 2015; Fitriani et al. 2014; Eri et al. 2013;

Ma'wa and Hoesain 2020; Liunokas et al. 2019; Laoh et al. 2013; Gassa 2011

Rahayu et al. 2021; Ardiana 2019; Armi et al.
2019; Rahmawati et al. 2019; de Barros et al. 2018; Rustam and Audina 2018; Warsa 2018; Nofia 2016; Saenong 2016; Isnaini et al. 2015; Kovendan et al. 2014; Owolabi et al. 2014; Chomsun and Muhfahroyin 2013; Fatmawati 2013; Leng and Reddy 2012; Hasna 2009; Kovendan et al. 2012; Legal et al. 1992

Chauhan et al. 2015

\begin{tabular}{|c|c|c|}
\hline \multirow[t]{3}{*}{$\begin{array}{l}\text { Jatropha } \\
\text { curcas L. }\end{array}$} & 1 & $\begin{array}{l}\text { Insect: Lepidoptera, Pyralidae, } \\
\text { Musca domestica, Callosobruchus } \\
\text { maculatus, Achaea Janata, Aphis } \\
\text { fabae, Sitophilus zeamais, } \\
\text { Rhyzorpertha dominica, Sitophilus } \\
\text { zeamais, Plutella xylostella), } \\
\text { Helicoverpa, Armigera, } \\
\text { Rhipicephalus(Boophilus) annulatus, } \\
\text { Spodoptera frugiperda, Homopteran } \\
\text { sp., Lepidopteran sp., and } \\
\text { Coleopteran sp., Aulacophora } \\
\text { foveicoltes, Liphaphis erysium, Musca } \\
\text { domestica }\end{array}$ \\
\hline & 4 & $\begin{array}{l}\text { Fungus: Aspergillus niger, } \\
\text { Aspergillus flavus, Colletotrichum } \\
\text { gloeosporioides, Pakospora } \\
\text { pachyrhizi, }\end{array}$ \\
\hline & 6 & Mollusca: Pomacea caniculata \\
\hline $\begin{array}{l}\text { Pangium edule } \\
\text { Reinw. }\end{array}$ & 1 & $\begin{array}{l}\text { Insect: Sitophylus oryzae, } \\
\text { Crocidolomia Binotalis, } \\
\text { Hypothenemus hampei, Spodoptera } \\
\text { exigua, Plutella xylostella, Spodoptera } \\
\text { litura, Aedes Aegypti. Coptotermes } \\
\text { gestroi, Scotinophora coarctata, } \\
\text { Plutella Xylostella, Blatella } \\
\text { Germanica, Schedorhinotermes sp }\end{array}$ \\
\hline
\end{tabular}

Shaqi 2021; Sutriadi et al. 2019; Ingle et al. 2017; Jide-Ojo et al. 2013; Devappa et al. 2012; Juliet et al. 2012; Nabil and Yasser 2012; Silva et al. 2012; Abdoul Habou et al. 2011; Tukimin et al. 2010; Adebowale and Adedire 2006; Li et al. 2006

Rahman et al. 2011; Heriyanto 2015

Banjarnahor et al. 2016

Kuntorini and Rusmiati 2021; Yulianti 2021; Hutasoit et al. 2020; Tampil et al. 2020; Manoppo et al. 2019; Haq and Desyanti 2018; Verawati 2018; Noerfitryani 2017; Sakul 2017; Hidayat 2016; Rusda 2014; Wiryadiputra et al. 2014; Sakul et al. 2012; Salaki et al. 2012; Yuningsih and Kartina 2007 
Afrianto, WF et al. (2022). Jurnal Biologi Tropis, 22 (1): 220 - 243

DOI: http://dx.doi.org/10.29303/jbt.v22i1.3266

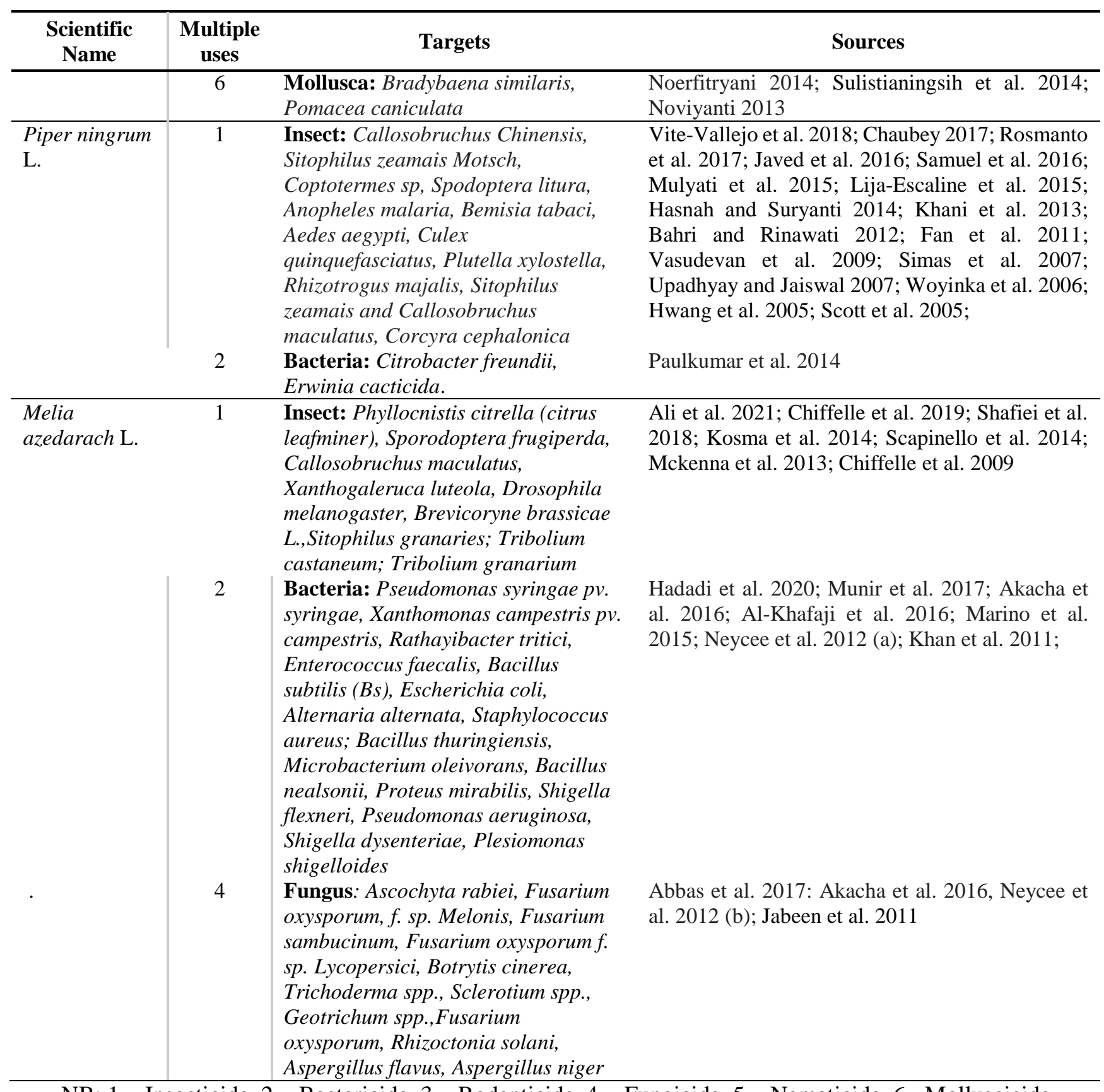

NB: $1=$ Insecticide, $2=$ Bactericide, $3=$ Rodenticide, $4=$ Fungicide, $5=$ Nematicide. $6=$ Molluscicide

\section{Regulation in Indonesia}

In Indonesia, the registration and regulation of pesticides must follow the government regulations (PP) of the Minister of Agriculture of the Republic of Indonesia Number 43 of 2019 concerning pesticides. Botanical pesticides were categorized as natural pesticides because they have active ingredients derived from living things. Natural pesticides have to assess the same as synthetic pesticides, tested for quality, formula, environmental fate, efficacy, and toxicity.

\section{Market potential}

Recently, the global market of botanical pesticides is only $2 \%$ of the pesticide production or over 3,000 tons per year (Kumar and Singh 2015). However, according to Damalas and Koutroubas (2018), botanical pesticides' value has been estimated at $\$ 3$ billion global, and it is predicted to increase significantly in the late 2040 and early 2050s (Olson 2015). The market is expanding because of the increasing demand for pesticide-free for growing organic food.

There are three factors to be considered to enlarge the market size. Firstly, the price should be regarded as an essential factor. This factor is influenced by the quality, geographic area, 
source (Isman 2016; Pratiwi and Nurlaeni 2021). Secondly, sustainability productivity for natural resources is one of the challenges (Hidayat et al. 2021). The productivity of biopesticides is still not optimal because of cultivation limited. Lastly, branding is also an essential factor, primarily the education for farmers because commonly, farmers are lack education and only focus on fast results.

\section{Conclusions}

In conclusion, based on the ethnobotany of the Indonesian community, 149 plant species can be used as insecticide, bactericide, rodenticide, fungicide, nematicide, and molluscicide and utilized as an alternative to synthetic/chemical pesticides for pest management. These species were dominated by the Compositae (14 species) and mainly used from the leaf part. The result of this study can be used as database information for further research regarding the efficacy, formulation, and conservation strategies for sustainable management. Furthermore, collecting the data can encourage citizens to be involved, so there is a bridge between people and nature and increasing community utilization (Afrianto and Najah 2017).

\section{Acknowledgment}

We thank all reviewers for their valuable comments.

\section{References}

Abbas G., Kashif M., Khan T. A., Bhatti H. A., Haque S., Naqvi S., \& Farooq A. D. (2018). Cytotoxic, embryotoxic, Insecticidal and Anti-Microbial Activities of Standardized Areca Catechu Nut. Pakistan Journal of Pharmaceutical Sciences, 31 (2): 385-392.

Abbas M. K., Ahmad M., Barkat K., \& Aslam N. (2017). Antifungal, Antioxidant and Phytochemical Screening of Melia Azedarach Flower Extracts by Using Different Solvents. Journal of Pharmaceutical Research International, 20 (1): 1-12.

Abdoul-Habou Z., Haougui A., Mergeai G., Haubruge E., Toudou A., \& Verheggen F. (2011). Insecticidal Effect of Jatropha curcas Oil on The Aphid Aphis fabae (Hemiptera: Aphididae) and on The Main
Insect Pests Associated with Cowpeas (Vigna unguiculata) in Niger. Tropicultura, 29 (4): 225-229.

Acero, L. H. (2019). Insecticidal Property of Jackfruit (Artocarpus heterophyllus) Peel Ethanol Extract Against Rice Weevils (Sitophilus oryzae). International Journal of Bioscience, Biochemistry and Bioinformatics, 158 (9): 158-165.

Achmad, A \& Suryana I. (2015). Testing The Activity of Betel Leaf Extract (Piper betle Linn.) against Rhizoctonia sp. In Vitro. Buletin Penelitian Tanaman Rempah dan Obat, 20 (1): 92-98.

Afrianto, W. F., \& Najah, S. K. (2017). Peran Citizen Science dalam Upaya konservasi Biodiversitas. Prosiding Semnas Biodiversitas, 6(1), 162-164.

Afrianto, W. F., Hikmat, A., \& Widyatmoko, D. (2017). Growth and habitat preference of Acacia decurrens Willd. (Fabaceae) after the 2010 eruption of Mount Merapi, Indonesia. Asian Journal of Applied Sciences, 5(1): 65-72.

Afrianto W. F, Tamnge F., Hidayatullah T., \& Hasanah L. N. (2021). Local Knowledge of Plant-Based Nutrition Sources from Forgotten Foods in Datengan Village, East Java, Indonesia. Asian Journal of Ethnobiology, 4 (1): 53-64.

Afrianto W. F., Tamnge F., \& Hasanah L. N. (2020). A Relation Between Ethnobotany and Bioprospecting of Edible Flower Butterfly Pea (Clitoria ternatea) in Indonesia. Asian Journal of Ethnobiology, 3 (2): 51-61.

Al-Khafaji N. J., Al-Zubaedi R. M., \& Al-Azawi S. J. (2016). Evaluation of Antibacterial Effects of Melia azedarach Fruit Extracts Against Some Isolated Pathogenic Bacteria. Veterinary Science Development, 6 (1): 1-3.

Ali K., Sagheer M., ul Hasan M., Rashid A., \& Shahid M. (2021). Bioactivity of Medicinal Plant Extracts as Toxicants and Enzyme Inhibitors Against Insect Pests of Stored Commodities. Journal of Crop Protection, 10 (1): 95-109.

Anderson T. A., Salice C. J., Erickson R. A., McMurry S. T., Cox S. B., Smith L. M. (2013). Effects of Landuse and Precipitation on Pesticides and Water Quality in Playa Lakes of The Southern High Plains. Chemosphere, 92 (1): 84-90. 
Andrés M. F., González-Coloma A., Sanz J., Burillo J., \& Sainz P. (2012). Nematicidal Activity of Essential Oils: a Review. Phytochemistry Reviews, 11 (4): 371-390.

Anisah, A. \& Sukesi T. W. (2018). Test The Effectiveness of Betel Leaf Extract (Piper betle L.) as Larvate Larvae of House Flies (Musca domestica). Jurnal Vektor Penyakit, 12 (1): 39-46.

Apiaceae A., Cupressaceae G., \& Lamiaceae L. (2011). Iranian Plant Essential Oils as Sources of Natural Insecticide Agents. International Journal of Biological Chemistry, 5 (5): 266-290.

Arambewela L., Kumaratunga K. G., \& Dias K. (2010). Studies on Piper betle of Sri Lanka. J Natn Sci Foundation Sri Lanka, 33 (2):133-9.

Ardiana, I. (2019). Utilization of noni fruit extract (Morinda citrifolia L.) as a botanical pesticide for pest control of Spodoptera litura F. in Palm (Brassica juncea L.). Unpublished undergraduate thesis in partial fulfillment of the requirements for the degree of Bachelor, Jenderal Soedirman University, Purwokerto, Central Java, Indonesia.

Ariyanti E., Jahuddin R., \& Yunus M. (2012). Potential of Betel Leaf Extract (Piper betle Linn) as a Biofungicide for Strawberry (Collectotrichum fragarie Brooks) Rot Disease In Vitro. Agroteknos 2 (3): 150155.

Bahri, S. \& Rinawati R. (2012). Terpenoid Compounds Isolated from Pepper Leaves (Piper Nigrum, Linn) and Their Bioactivity Test Against Pests Callosobruncus Chinensis. Jurnal Sains MIPA Universitas Lampung, 3 (3): 158166.

Banjarnahor I., Wibowo L., Hariri A. M., \& Hasibuan R. (2016). Effect of Jatropha Seed Extract (Jatropha curcas L.) on Mortality of Golden Snail (Pomacea sp.) in Greenhouse. Jurnal Agrotek Tropika, 4 (2): 130-134.

Bassil K. L., Vakil C., Sanborn M., Cole D. C., Kaur J. S., \& Kerr K. J. (2007). Cancer Health Effects of Pesticides: Systematic Review. Canadian Family Physician, 53 (10): 1704-1711.

Beketov M. A., Kefford B. J, Schäfer RB, \& Liess M. (2013). Pesticides Reduce Regional Biodiversity of Stream
Invertebrates. Proceedings of the National Academy of Sciences 110 (27): 1103911043.

Belenguer V., Martinez-Capel F., Masiá A., \& Picó Y. (2014). Patterns of Presence and Concentration of Pesticides in Fish and Waters of The Júcar River (Eastern Spain). Journal of Hazardous Materials, 265: 271-279.

Brühl, C. A. \& Zaller J. G. (2019). Biodiversity Decline as a Consequence of an Inappropriate Environmental Risk Assessment of Pesticides. Frontiers in Environmental Science, 7 (177): 1-4.

Cavanagh, H. M. (2007). Antifungal Activity of The Volatile Phase of Essential Oils: a Brief Review. Natural Product Communications, 2 (12): 1297 - 1302.

Chaubey, M. K. (2017). Evaluation of Insecticidal Properties of Cuminum cyminum and Piper nigrum Essential Oils Against Sitophilus zeamais. Journal of Entomology, 14 (4): 148-154.

Chauhan N., Kumar P., Mishra S., Verma S., Malik A., \& Sharma S. (2015). Insecticidal Activity of Jatropha curcas Extracts Against Housefly, Musca domestica. Environmental Science and Pollution Research, 22 (19): 14793-14800.

Chiffelle I., Huerta A., Bobadilla V., Macuada G., Araya J. E., Curkovic T., \& Ceballos R. (2019). Antifeedant and Insecticidal Effects of Extracts from Melia azedarach Fruits and Peumus boldus Leaves on Xanthogaleruca luteola Larvae. Chilean Journal of Agricultural Research, 79 (4): 609-615.

Chiffelle I., Huerta F. A., \& Lizana R. D. (2009). Physical and Chemical Characterization of Melia azedarach L. Fruit and Leaf for Use as Botanical Insecticide. Chilean Journal of Agricultural Research, 69 (1): 38-45.

Chomsun, S. \& Muhfahroyin M. (2013). The Effect of Variations in Concentration of Noni (Morinda citrifolia) on Mortality of Pests of Pomacea caniculata L. as a Source of Biology Learning. Bioedukasi Jurnal Pendidikan Biologi, 4 (2): 1-8.

Clément, D. (1998). The Historical Foundations of Ethnobiology (1860-1899). Journal of ethnobiology, 18: 161-161.

Cita, K. D. (2020). Ethnobotany of Food Plant Used by Sundanese Ethnic in Kalaparea Village, Nyangkewok Hamlet, Sukabumi 
District, Indonesia. Asian Journal of Ethnobiology, 3 (1): 16-22.

Dadang. (1999). Source of Natural Insecticide: Training Materials, Development, and Utilization of Natural Insecticides. Bogor, Indonesia.

Damalas, C. A. \& Koutroubas S. D. (2018). Current Status and Recent Developments in Botanical Pesticides Use. Agriculture, 8 (1): 1-6.

Daswito R., Folentia R., \& MF M. Y. (2019). The Effectiveness of Green Betel Leaf Extract (Piper betle) as a Vegetable Insecticide Against Mortality of The House Fly (Musca domestica). Jurnal Kesehatan Terpadu (Integrated Health Journal), 10 (2): 44-50.

de Barros R. P., Reis L. S., da Costa J. G., Cunha A. L., Magalhaes I. C. S., da Silva C. G., dos Santos A. F., das Neves J. D. S., Duarte A. G., de Mello G. S. V., de Freitas J. D., dos Santos S. J., \& Franco S. P. B. (2018). Bioactivity and Phenolic Composition of Extracts of Noni (Morinda citrifolia L., Rubiaceae) in Tomato Moth (Tuta absoluta Meyrick, 1917) (Lepidoptera: Gelechiidae). African Journal of Agricultural Research, 13 (39): 2063-2069.

Devappa R. K., Angulo-Escalante M. A., Makkar H. P., \& Becker K. 2012. Potential of Using Phorbol Esters as an Insecticide Against Spodoptera frugiperda. Industrial Crops and Products, 38: 50-53.

Dewi M., Yuli A., Salbiah D., \& Sutikno A. (2017). Test of Several Concentrations of Areca Seed Flour (Areca catechu L.) Against Mortality of Larvae of Sweet Corn Cob Borer (Helicoverpa armigera Hubner). Unpublished undergraduate thesis in partial fulfillment of the requirements for the degree of Bachelor, Riau University, Riau, Indonesia.

Dudley N., Attwood S. J., Goulson D., Jarvis D., Bharucha Z. P., \& Pretty J. (2017). How Should Conservationists Respond to Pesticides as a Driver of Biodiversity Loss in Agroecosystems?. Biological Conservation, 209: 449-453.

Eka, N. (2021). Test the effectiveness of areca fruit seed extract (Areca Cetechu L.) as a natural insecticide against mortality of green leafhopper (Nephotettix virescens) Larvae D. Unpublished undergraduate thesis in partial fulfillment of the requirements for the degree of Bachelor, Raden Intan State Islamic University, Lampung, Indonesia.

Erawan T. S., Alilaj A. N., \& Iskandar J. (2018). Ethnobotany of Traditional Rituals in The Karangwangi Village, Cianjur District, West Java, Indonesia. Asian Journal of Ethnobiology, 1 (2): 53-60.

Eri E., Salbiah D., \& Laoh H. (2013). Test of Several Concentrations of Areca Nut Extract (Catechu Area) for Controlling Gray Caterpillar Pests (Spodoptera Liturra F.) on Mustard (Brassica Juncea L.). Unpublished undergraduate thesis in partial fulfillment of the requirements for the degree of Bachelor, Riau University, Riau, Indonesian.

Fadhillah, W. (2020). A Qualitative Test of Primary and Secondary Metabolites of Bintaro Plant as a Rat (Rattus argentiventer) Pest Repellent. International Journal of Environment, Agriculture and Biotechnology, 5 (5): 1300-1303.

Fan L. S., Muhamad R., Omar D., \& Rahmani M. (2011). Insecticidal Properties of Piper nigrum Fruit Extracts and Essential Oils Against Spodoptera litura. International Journal of Agriculture \& Biology, 13 (4): 517-522.

Fatmawati, Y. (2013). Effectiveness of noni (Morinda citrifolia L.) fruit extract as a botanical inseticide on corn pest mortality (Helicoverpa armigera Hubner). Unpublished undergraduate thesis in partial fulfillment of the requirements for the degree of Bachelor, University of Muhammadiyah Malang, Malang, East Java, Indonesia.

Firdaus, A. A. \& Kriswandana F. (2018). Potential of Jackfruit Leaf Extract as Biolarvicide for Culex sp. Gema Lingkungan Kesehatan, 16 (1): 14-20.

Fitriani M., Laoh J. H., \& Rustam R. (2014). Test various concentrations of betel nut extract (Areca catechu L.) to control the green ladybug (Nezara viridula L.) (Hemiptera: pentatomidae) in the laboratory. Unpublished undergraduate thesis in partial fulfillment of the requirements for the degree of Bachelor, Riau University, Riau, Indonesian.

Gaoue O. G., Coe M. A., Bond M., Hart G., Seyler B. C., \& McMillen H. (2017). Theories and Major Hypotheses in 
Ethnobotany. Economic Botany, 71 (3): 269-287.

Gassa, A. (2011). Effect of Areca Nut (Areca catechu) on Mortality of Golden Snail (Pomacea canaliculata) at Various Stages. J. Fitomedika, 7 (3): 171-174.

Geiger F., Bengtsson J., Berendse F., Weisser W. W., Emmerson M., Morales M. B., Ceryngier P., Liira J., Tscharntke T., Winqvist C., Eggers S., Bommarco R., Part T., Bretagnoile V., Plantegenest M., Clemen L. W., Dennis C., Paler C., Onate J. J., Guerrero I., Hawro V., Aavik T., Thies C., Flohre A., Hanke S., Fischer C., Goedhart P. W., \& Inchausti P. (2010). Persistent Negative Effects of Pesticides on Biodiversity and Biological Control Potential on European Farmland. Basic and Applied Ecology, 11 (2): 97-105.

Glare T. R., Gwynn R. L., \& Moran-Diez M. E. (2016). Development of Botanical Pesticides and Future Opportunities. Microbial-Based Botanical Pesticides, 211-221.

Gobai M., Tobing O. L., \& Rochman N. (2015). Insecticidal Power of Otikai Leaf Extract (Alphitonia sp.) and Betel Nut Extract (Areca catechu L.) on The Mortality Rate of The Warehouse Pest Insect Callosobruchus chinensis L. Jurnal Agronida, 1 (2): 71-82.

Guo Y., Chen J., Ren D., Du B., Wu L., Zhang Y., Wang Z., \& Qian S. (2021). Synthesis of Osthol-Based Botanical Fungicides and Their Antifungal Application in Crop Protection. Bioorganic \& Medicinal Chemistry, 40: 116184.

Gupta V., Tripathi M., \& Khanb N. (2015). Characterization of Cysteine Protease Inhibitor from Artocarpus Heterophyllus and Cry Protein from Bt (Bacillus Thuringiensis) for Antimetabolic Activity of Scirpophage incertulas (Yellow Stem Borer). Annals of Plant and Soil Research, 17: 258-263.

Guswenrivo I., Tarmadi D., \& Yusuf S. (2013). Insecticidal Activity of Bintaro Fruit Extract (Cerbera manghas) Against Rice Lice Sitophilus oryzae (Coleoptera: Curculionidae). Jurnal Ilmu dan Teknologi Kayu Tropis, 11 (1): 82-89.

Hadadi Z., Nematzadeh G. A., \& Ghahari S. 2020. A study on The Antioxidant and Antimicrobial Activities in the Chloroformic and Methanolic Extracts of
6 Important Medicinal Plants Collected from North of Iran. BMC Chemistry, 14 (1): $1-11$

Hans, A. L \& Saxena S. 2021. Plant Bioprospecting for Biopesticides and Bioinsecticides. John Wiley \& Sons Inc, West Sussex.

Hasnah, M. R. \& Suryanti L. (2014). Efficacy of Black Pepper Powder in Controlling The Pest Sitophilus zeamais on Corn Kernels During Storage. Jurnal Penelitian Universitas Jambi Seri Sains, 16 (2): 2332.

Hasnah, H. (2009). The Effectiveness of Noni Fruit Extract (Morinda citrifolia L.) on Mortality of Plutella xylostella L. in Mustard Plants. Jurnal Floratek, 4 (1): 2940.

Haq, K. D \& Desyanti Z. (2018). Using a Pesticide Extracted from Leaves of Simaung (Pangium edule Reinw.) for Control of Soil Termite Pest (Schedorhinotermes sp.) as One of Solution Conservation. In Forschler B. T., Lie H. F., Foo F. K., Yusuf S., \& Himmi $\mathrm{S}$. K., Ismayati $\mathrm{M}$ (eds). In The 12th Conference of The Pacific Rim Termite Research Group, March 21-22, Grand Inna Malioboro, Yogyakarta.

Haryanta, D. \& Joeniarti E. (2021). Potential Test of Leaf Extract of Bintaro (Cerbera mangos) as a Botanical Insecticide Against Spodoptera litura F. Agrin 25 (1): 10-21.

Hayata, H. (2017). Pest Response of Lasioderma Serricorne to The Formulated Phosphine (Tablets and Bags) in Areca Nuts. Jurnal Ilmiah Universitas Batanghari Jambi, 14 (4): 87-92.

Heriyanto, H. (2015). Study of Rest Disease Control in Soybean with Botanical Pesticides. Jurnal Ilmu-Ilmu Pertanian, 21 (1): 46-55.

Hidayat S., Zuhud E. A., Widyatmoko D., Bahruni B., \& Batubara I. (2021). The Commercial Potential of Forest Trees as Medicinal and Health Ingredients. Biodiversitas Journal of Biological Diversity, 22 (7): 2795-2804.

Hidayat, S. (2016). Use of Kluwek Seed Extract (Pangium Edule Reinw.) as a Botanical Insecticide Against Mortality (Blatella Germanica L.). Sainmatika: Jurnal Ilmiah Matematika dan Ilmu Pengetahuan Alam, 11(1): 14-19. 
Hikal W. M., Baeshen R. S., \& Said-Al Ahl H. A. H. (2017). Botanical Insecticide as Simple Extractives for Pest Control. Cogent Biology, 3 (1): 1-16.

Hurtada, J. M. U. P. A., Divina B. P., \& Ducusin R. J. T. (2014). Anthelmintic Efficacy of Jackfruit (Artocarpus heterophyllus L.) and Tamarind (Tamarindus indica L.) Leaves Decoction Against Gastrointestinal Nematodes of Goats. Philippine Journal of Veterinary and Animal Sciences, 38 (2): 157-166.

Hutasoit H., Rompas C. F., \& Manoppo J. S. S. (2020). Potency of Bioinsecticide Extract of Pangi Seed (Pangium edule, Reinw) in Pest Control of Onion Caterpillar (Spodoptera exigua Hubner). Nukleus Biosains, 1 (2): 61-69.

Hwang K. S., Kim Y. K., Park K. W., \& Kim Y. T. (2017). Piperolein B and Piperchabamide D Isolated from Black Pepper (Piper nigrum L.) as Larvicidal Compounds Against The Diamondback Moth (Plutella xylostella). Pest Management Science, 73(8): 1564-1567.

Ingle K. P., Deshmukh A. G., Padole D. A., Dudhare M. S. (2017). Screening of Insecticidal Activity of Jatropha Curcas (L.) against Diamond Back Moth and Helicoverpa Armigera. Seed, 5 (80): 4450.

Irtiawati I., Rafdinal R., \& Wardoyo E. R. P. (2020). Etnobotany Utilization of Toxic Plants in Keranji Paidang Village, Sengah Temila District, Landak Regency. Jurnal Protobiont, 9 (2): 132-141.

Iskandar, J. (2017). Ethnobiology and Cultural Diversity in Indonesia. Umbara, 1 (1): 27 42.

Iskandar, J. \& Iskandar B. S. (2017). Various Plants of Traditional Rituals: Ethnobotanical Research Among The Baduy Community. Biosaintifika: Journal of Biology \& Biology Education, 9 (1): 114-125.

Isman, M. B. (2016). Pesticides Based on Plant Essential Oils: Phytochemical and Practical Considerations. J. Am. Chem. Soc, 13-26.

Isman, M. B. \& Grieneisen M. L. (2014). Botanical Insecticide Research: Many Publications, Limited Useful Data. Trends in Plant Science, 19 (3): 140-145.

Isnaini M., Pane E. R., \& Wiridianti S. (2015). Testing of Several Types of Botanical
Insecticides Against Rice Lice (Sitophilus Oryzae L). Jurnal Biota, 1(1): 1-8.

Jabeen K., Javaid A., Ahmad E., \& Athar M. (2011). Antifungal Compounds from Melia azedarach Leaves for Management of Ascochyta rabiei, the Cause of Chickpea Blight. Natural Product Research, 25 (3): 264-276.

Jadid N., Kurniawan E., Himayani C. E. S., Prasetyowati I., Purwani K. I., Muslihatin W., Hidayati D., \& Tjahjaningrum I. T. D. (2020). An Ethnobotanical Study of Medicinal Plants Used by The Tengger Tribe in Ngadisari Village, Indonesia. Plos one, 15 (7): e0235886.

Jankowska M., Rogalska J., Wyszkowska J., \& Stankiewicz M. 2018. Molecular Targets for Components of Essential Oils in The Insect Nervous System-a Review. Molecules, 23 (1): 1-20.

Javed M., Majeed M. Z., Arshad M., Ahmad M. H., \& Ghafoor H. A. (2016). Insecticidal Potentiality of Eruca sativa (mill.), Piper nigrum (1.) and Withania somnifera (1.) Extracts Against Trogoderma granarium (everts) (Coleoptera: Dermestidae). Int J Fauna Biol Stud, 3 (1): 18-20.

Jide-Ojo C., Gungula D. T., \& Ojo O. O. (2013). Extracts of Jatropha curcas L. Exhibit Significant Insecticidal and Grain Protectant Effects Against Maize Weevil, Sitophilus zeamais (Coleoptera: Curculionidae). Journal of Stored Products and Postharvest Research, 4 (3): 44-50.

Juliet S., Ravindran R., Ramankutty S. A., Gopalan A. K. K., Nair S. N., Kavillimakkil A. K., Bandyopadhyay A., Rawat A. K. S., \& Ghosh S. (2012). Jatropha curcas (Linn) Leaf Extract-a Possible Alternative for Population Control of Rhipicephalus (Boophilus) annulatus. Asian Pacific Journal of Tropical Disease, 2 (3), 225-229.

Kewa, K. (2019). Toxicity of betel nut (Areca catechu Linn.) seed extract on mortality of fruit flies (Bactrocera carambolae Linn.). Unpublished undergraduate thesis in partial fulfillment of the requirements for the degree of Bachelor, Atma Jaya Yogyakarta University, Yogyakarta, Indonesia.

Khan A. V., Ahmed Q. U., Mir M. R., Shukla I., \& Khan A. A. (2011). Antibacterial Efficacy of the Seed Extracts of Melia 
azedarach Against Some Hospital Isolated Human Pathogenic Bacterial Strains. Asian Pacific Journal of Tropical Biomedicine, 1(6): 452-455.

Khani M., Awang R. M., Omar D., \& Rahmani M. (2013). Toxicity, Antifeedant, Egg Hatchability and Adult Emergence Effect of Piper nigrum L. and Jatropha curcas L. Extracts Against Rice Moth, Corcyra cephalonica (Stainton). Journal of Medicinal Plants Research, 7 (18): 12551262.

Kim K. H., Kabir E., \& Jahan S. A. (2017). Exposure to Pesticides and The Associated Human Health Effects. Science of The Total Environment, 575: 525-535.

Kosma P., Romeo B., Bouba D., Armand A. B., \& Augustin G. (2014). Bioefficacity of The Powder of Melia azedarach Seeds and Leaves Against Callosobruchus maculatus, on Cowpea Seeds (Vigna unguiculata) in Storage. E3 Journal of Agricultural Research and Development, 5 (4): 072-078.

Kovendan K., Shanthakumar S. P., Praseeja C., Kumar P. M., Murugan K., \& Vincent S. 2014. Mosquitocidal Properties of Morinda citrifolia L. (Noni) (Family: Rubiaceae) Leaf Extract and Metarhizium anisopliae Against Malaria Vector, Anopheles stephensi Liston. (Diptera: Culicidae). Asian Pacific Journal of Tropical Disease, 4: S173-S180.

Kovendan K., Murugan K., Shanthakumar S. P., Vincent S., \& Hwang J. S. (2012). Larvicidal Activity of Morinda citrifolia L. (Noni) (Family: Rubiaceae) Leaf Extract Against Anopheles stephensi, Culex quinquefasciatus, and Aedes aegypti. Parasitology Research 111 (4): 1481-1490.

Kumar, S. \& Singh A. (2015). Botanical Pesticidess: Present Status and The Future Prospects. J Fertil Pestic 6 (2): 100-129.

Kumarasingha S. P., Ihalamulla R. L., Arambewela L. S. R., \& Dissanayake D. S. (2002). Larvicidal Effects of Mineral Turpentine, Low Aromatic White Spirits, Aqueous Extracts of Cassia Alata and Aqueous Extracts, Ethanolic Extracts and Essential Oil of Betel Leaf (Piper betle) on Chrysomya megacephala. Int J Dermatol, 41: 877-80.

Kuntorini, E. M. \& Rusmiati R. (2021). Effect of Kepayang Leaf Extract (Pangium edule
Reinw.) as a Botanical Pesticide on Mortality of Caterpillar (Spodoptera litura F.) Mortality. Bioscientiae, 17 (2): 77-87.

Laoh H., Rustam R., \& Permana R. (2013). Several Doses of Local Riau Betel Nut Flour (Areca catechu L.) to Control Golden Snail (Pomacea canaliculata L.) Pests on Rice. PEST Tropical Journal, 1 (2): 1-8.

Legal L., David J. R., \& Jallon J. M. (1992). Toxicity and Attraction Effects Produced by Morinda citrifolia Fruits on The Drosophila melanogaster Complex of Species. Chemoecology, 3 (3): 125-129.

Leng, P. H. \& Reddy G. V. (2012). Bioactivity of Selected Eco-Friendly Pesticides Against Cylas formicarius (Coleoptera: Brentidae). Florida Entomologist, 10401047.

Lija-Escaline J., Senthil-Nathan S., Thanigaivel A., Pradeepa V., Vasantha-Srinivasan P., Ponsankar A., Edwin E. S., Selin-Rani S., \& Abdel-Megeed. (2015). Physiological and Biochemical Effects of Botanical Extract from Piper nigrum Linn (Piperaceae) Against The Dengue Vector Aedes aegypti Liston (Diptera: Culicidae). Parasitology research, 114 (11): 42394249.

Li J., Wu F. H., Chen Y. Y., \& Chen F. (2006). Insecticidal Activity of Jatropha curcas Seed Extracts Against Several Insect Pest Species. Pesticides Shenyang, 45 (1): 57.

Liunokas A. B., Bana J. J., \& Amalo D. (2019). The Effect of Areca Nut Extract (Areca Catechu L.) on The Survival of Golden Snail Eggs (Pomacea Canaliculata Lamarck). Jurnal Biologi Tropis, 19 (2): 294-301.

Lorenza, I. (2019). Tests of some concentrations of areca seed extract (Areca Catechu L.) in controlling the pest of the horn better (Oryctes rhinoceros) in palm oil plant. Unpublished undergraduate thesis in partial fulfillment of the requirements for the degree of Bachelor, Sultan Syarif Kasim State Islamic University, Riau, Indonesia.

Ma'wa, N. \& Hoesain M. (2020). Effect of Concentration of Neem and Areca Nut Extract on Mortality of Golden Snail (Pomacea canaliculata L.). Jurnal Proteksi Tanaman Tropis, 1 (1): 9-13.

Mading M., Kazwaini M., Utomo B., Arwati H., \& Yotopranoto S. (2018). Effects of Areca 
catechu L. Seed Extract on Mortality Anopheles vagus Larvae. Kemas: Jurnal Kesehatan Masyarakat, 13 (3): 366-373.

Magierowicz K., Górska-Drabik E., \& Sempruch C. (2019). The Insecticidal Activity of Satureja Hortensis Essential Oil and Its Active Ingredient-Carvacrol Against Acrobasis advenella (Zinck.) (Lepidoptera, Pyralidae). Pesticide Biochemistry and Physiology, 153: 122128.

Manoppo J. S. S., Sakul E. H., \& Tengker A. C. (2019). Potential of Bioinsecticide from Leaf Extract, Bark and Seed of Pangi (Pangium edule reinw.) in Increasing Mortality of Crocidolomia Binotalis Larvae. Frontiers: Jurnal Sains dan Teknologi, 2 (1): 9-19.

Mardiastuti A., Masy'ud B., Ginoga L. N., \& Sastranegara H. 2021a. Overview of the Traditional Indonesian Knowledge on the Use of Reptiles. The 1st Journal of Environmental Science and Sustainable Development Symposium, Sept. 28-30, Jakarta, Indonesia.

Mardiastuti A., Masy'ud B., Ginoga L. N., \& Sastranegara H. 2021b. Describing and Visualizing the Progress of Ethnozoology in Indonesia by Using VOSviewer. 2nd International Symposium on Transdisciplinarity Approach for Knowledge Co-Creation in Sustainability Understanding Complexity and Transdisciplinarity for Environmental Sustainability, Nov. 3-4, Bogor, Indonesia.

Marino G., Gaggia F., Baffoni L., Toniolo C., \& Nicoletti M. (2015). Antimicrobial Activity of Melia azedarach Fruit Extracts for Control of Bacteria in Inoculated InVitro Shoots of 'MRS 2/5' Plum Hybrid and Calla Lily and Extract Influence on The Shoot Cultures. European Journal of Plant Pathology, 141 (3): 505-521.

Mawardi M., Elfrida E., \& Fitri R. (2018). Effect of Jengkol Peel Extract and Sri Fortune Leaves on Mortality of Pomecea canaliculata. Jurnal Jeumpa, 5 (1): 56-64.

Mckenna M. M., Hammad E. M. A., \& Farran M. T. (2013). Effect of Melia azedarach (Sapindales: Meliaceae) Fruit Extracts on Citrus Leafminer Phyllocnistis citrella (Lepidoptera: Gracillariidae). Springer Plus, 2 (1): 1-6.

Meftaul I. M., Venkateswarlu K., Dharmarajan R., Annamalai P., \& Megharaj M. (2020).
Pesticides in The Urban Environment: A Potential Threat that Knocks at The Door. Science of The Total Environment, 711: 134612.

Mingo V., Lötters S., \& Wagner N. (2016). Risk of Pesticide Exposure for Reptile Species in The European Union. Environmental Pollution, 215: 164-169.

Mohottalage S., Tabacchi R., \& Guerin P. M. (2007). Components from Sri Lankan Piper betle L. Leaf Oil and Their Analogues Showing Toxicity Against the Housefly, Musca domestica. Flavour and Fragrance Journal, 22 (2): 130-138.

Mulyati S., Jayuska A., \& Ardiningsih P. (2015). Activities of Pepper Leaf Essential Oil (Piper nigrum L.) Against Termites Coptotermes sp. Jurnal Kimia Khatulistiwa, 4 (3): 100-106.

Munir T., Mohyuddin A., Khan Z., \& Haq R. (2017). Exploration of Antibacterial Potential of Melia azedarach L. Scientific Inquiry and Review 1 (1): 19-26.

Nabil, A. A. \& Yasser A. M. K. (2012). Jatropha curcas Oil as Insecticide and Germination Promoter. Journal of Applied Sciences Research, 668-675.

Nahdi M. S., Martiwi I. N. A, \& Arsyah D. C. (2016). The Ethnobotany of Medicinal Plants in Supporting The Family Health in Turgo, Yogyakarta, Indonesia. Biodiversitas Journal of Biological Diversity, 17 (2): 900-906.

Nair, S. S. \& Kavrekar V. (2017). In Vitro Screening of Larvicidal and Insecticidal Activity of Methanolic Extracts of Artocarpus heterophyllus, Artocarpus altilis and Piper betle. International Journal of Environment, Agriculture and Biotechnology, 2 (1): 238672.

Navia Z. I., Audira D., Afifah N., Turnip K., Nuraini N., \& Suwardi A. B. (2020). Ethnobotanical Investigation of Spice and Condiment Plants Used by the Taming Tribe in Aceh, Indonesia. Biodiversitas Journal of Biological Diversity, 21 (10): 4467-4473.

Neycee M. A., Nematzadeh G. H. A., Dehestani A., \& Alavi M. (2012). Evaluation of Antibacterial Effects of Chinaberry (Melia azedarach) Against Gram-Positive and Gram-Negative Bacteria. Int J Agric Crop Sci, 4 (11): 709-712.

Neycee M. A., Nematzadeh G. H. A, Dehestani A., \& Alavi M. (2012). Assessment of 
Antifungal Effects of Shoot Extracts in Chinaberry (Melia azedarach) Against 5 Phytopatogenic Fungi. International Journal of Agricultural and Crop Sciences, 4: 474-477.

Noerfitryani, N. (2017). Kluwak (Pangium edule Reinw) seed Extract as Ovicide in Golden Snail Egg (Pomacea canaliculata L.). Journal Tabaro Agriculture Science, 1 (2): 78-85.

Nofia, D. (2016). Activity of noni extract (Morinda citrifolia L.) (Rubiaceae) against Crocidolomia pavonana (F.) (lepidoptera: crambidae). Unpublished undergraduate thesis in partial fulfillment of the requirements for the degree of Bachelor, Andalas University, Padang, West Sumatera, Indonesia.

Ntzani E. E., Ntritsos G. C. M., Evangelou E., \& Tzoulaki I. (2013). Literature Review on Epidemiological Studies Linking Exposure to Pesticides and Health Effects. EFSA Supporting Publications 10 (10): 497E.

Noviyanti, R. D. (2013). Test the effectiveness of kluwek (Pangium edule, Reinw) on mortality of snails (Bradybaena similaris). Unpublished undergraduate thesis in partial fulfillment of the requirements for the degree of Bachelor, Sunan Gunung Djati State Islamic University, Bandung, West Java, Indonesia.

O’Bryan C. A., Pendleton S. J., Crandall P. G., \& Ricke S. C. (2015). Potential of Plant Essential Oils and Their Components in Animal Agriculture-In Vitro Studies on Antibacterial Mode of Action. Frontiers in Veterinary Science, 2 (35): 1-8.

Olson, S. (2015). An Analysis of The Botanical Pesticides Market Now and Where is Going. Outlooks Pest Manag, 26: 203206.

Owolabi M. S., Padilla-Camberos E., Ogundajo A. L., Ogunwande I. A., Flamini G., Yusuff O. K., Allen K., Flores-Fernandez K. I., \& Flores-Fernandez J. M. (2014). Insecticidal Activity and Chemical Composition of the Morinda Lucida Essential Oil Against Pulse Beetle Callosobruchus maculatus. The Scientific World Journal, 1-7.

Pakki T., Taufik M., \& Adnan A. M. (2009). Study On the Potential of Botanical Rodenticides from Jengkol Seeds for Control of Rat Pests in Corn. Prosiding
Seminar Nasional Serealia: 378-382, Indonesia.

Pandey, A. K. \& Tripathi Y. C. (2017). Ethnobotany And Its Relevance in Contemporary Research. J Med Plants Stud, 5 (3): 123-9.

Park M. G., Blitzer E. J., Gibbs J., Losey J. E., \& Danforth B. N. (2015). Negative Effects of Pesticides on Wild Bee Communities can be Buffered by Landscape Context. Proceedings of the Royal Society B: Biological Sciences 282(1809): 20150299.

Paulkumar K., Gnanajobitha G., Vanaja M., Rajeshkumar S., Malarkodi C., Pandian K., \& Annadurai G. (2014). Piper nigrum Leaf and Stem Assisted Green Synthesis of Silver Nanoparticles and Evaluation of Its Antibacterial Activity Against Agricultural Plant Pathogens. The Scientific World Journal, 1-9.

Pavela, R. \& Benelli G. (2016). Essential Oils as Ecofriendly Botanical Pesticidess? Challenges and Constraints. Trends in Plant Science, 21 (12): 1000-1007.

Peixoto M. G., Costa-Júnior L. M., Blank A. F., da Silva Lima A., Menezes T. S. A., de Alexandria Santos D., Alves P. B., de Holanda Cavalcanti S. C., Bacci L., \& de Fátima Arrigoni-Blank M. (2015). Acaricidal Activity of Essential Oils from Lippia alba Genotypes and Its Major Components Carvone, Limonene, and Citral Against Rhipicephalus microplus. Veterinary Parasitology, 210 (1-2): 118-122.

Pimentel, D. \& Burgess M. (2014). Environmental and Economic Costs of the Application of Pesticides Primarily in the United States. In Integrated Pest Management, Springer, Dordrecht.

Prance, G. T. (1991). What is Ethnobotany Today?. Journal of Ethnopharmacology, 32 (1-3): 209-216.

Pratiwi, R. A. \& Nurlaeni Y. (2021). The Potency of Myrtaceae Family from Cibodas Botanic Gardens (Cianjur, Indonesia) as Botanical Pesticide. Biodiversitas Journal of Biological Diversity, 22 (10): 46484664.

Purwani K. I., Wijayawati L., Nurhatika S., Sa'Diyah N. A., \& Arifiyanto A. (2014). Bintaro (Cerbera odollam) Leaf Extract as a Potential Biological Pest Control Toward Spodopteralitura F. Mortality. J. Appl. Environ. Biol. Sci, 4 (4): 18-23. 
Putri L. S. E., Dasumiati, Kritiyanto, Mardiansyah, Malik C., Leuvinadrie L. P., \& Mulyono E. A. (2016). Ethnobotanical Study of Herbal Medicine in Ranggawulung Urban Forest, Subang District, West Java, Indonesia. Biodiversitas Journal of Biological Diversity, 17 (1): 172-176.

Rahayu R., Herwina H., \& Jannatan R. (2021). Efficacy of Noni (Morinda citrifolia L.) Ethanolic Leaf Extract Against German Cockroach (Blattella germanica L.). Pakistan Journal of Biological Sciences: PJBS, 24 (5): 629-635.

Rahman M., Ahmad S. H., Mohamed M. T. M., \& Rahman M. Z. A. (2011). Extraction of Jatropha curcas Fruits for Antifungal Activity Against Anthracnose (Colletotrichum gloeosporioides) of Papaya. African Journal of Biotechnology, 10 (48), 9796-9799.

Rahmawati, A. (2020). The Effectiveness of Green Betel Leaf Extract (Piper betle L.) as a Natural Insecticide Against The Mortality of Green Grasshoppers (Oxya serville). Pedagogos: Jurnal Pendidikan, 2 (2): 61-65.

Rahmawati D., Djamilah D., \& Simanihuruk B. W. (2019). Effect of Noni Fruit Extract (Morinda citrifolia L.) and Application Time to Control Crocidolomia binotalis Zell. on Cabbage Plant. Akta Agrosia, 22 (1): 13-21.

Ramadhanti, D. (2020). The effectiveness of noni seed flour (Morinda citrifolia L) on the control of root chole nematodes (Meloidogyne spp.) on tomato (Lycopersicum esculetum Mill.). Unpublished undergraduate thesis in partial fulfillment of the requirements for the degree of Bachelor, Sultan Syarif Kasim Islamic State University, Riau, Indonesian.

Ratnani D. A. S., Junitha I. K., Kriswiyanti E., \& Dhana I. N. (2021). The Ethnobotany of Ngusaba Ceremonial Plant Utilization by Tenganan Pegringsingan Community in Karangasem, Bali, Indonesia. Biodiversitas Journal of Biological Diversity, 22(4): 2078-2087.

Riah W., Laval K., Laroche-Ajzenberg E., Mougin C., Latour X., \& TrinsoutrotGattin I. (2014). Effects of Pesticides on Soil Enzymes: a Review. Environmental Chemistry Letters, 12 (2): 257-273.
Rikardo, K. \& Nuryasin N. (2018). Toxicity of Betel Nut Extract (Areca catechu L.) To Cabbage Crop Caterpillar (Crocidolomia pavonana F.) in The Laboratory. Jurnal Agrotek Tropika, 6 (1): 44-49.

Rosmanto R., Sutikno A., \& Salbiah D. (2017). Test of Multiple Doses of Black Pepper Seed Flour (Piper nigrum L.) for Pest Control Callosobruchus Chinensis L. On Green Bean Seeds in Storage. Jurnal Sagu, 15 (2): 21-30.

Rusda, I. (2014). The effectiveness of picung (Pangium edule Reinw) against the mortality of the coffee fruit bear (Hypothenemus hampei Ferrari). Unpublished undergraduate thesis in partial fulfillment of the requirements for the degree of Bachelor, Jember University, Jember, East Java, Indonesia.

Rustam, R. \& Audina M. (2018). Test of Noni (Morinda citrifolia L.) Flour Against Corn Powder Pest Sitophilus zeamais M. (Coleoptera; Curculionidae). Jurnal Agroekoteknologi, 10 (1): 80-93.

Saenong, M. S. (2016). Indonesian Plants are Potential as Botanical Insecticides to Control Corn Powder Beetles (Sitophilus Spp.). Jurnal Penelitian dan Pengembangan Pertanian, 35 (3): 131142.

Sakul, E. H. (2017). Impact of Botanical Insecticides Derived from Pangium edule Reinw. and Annona muricata L. Seed Extracts on the "Gay Gantung" Diamondback Moth, Plutella xylostella 1. Agrotech Journal, 2 (2), 27-35.

Sakul E. H., Manoppo J. S., \& Taroreh D., Gerungan R. I., \& Gugule S. 2012. Logong Beetle Pest Control (Sitophylus oryzae L.) by Using Pangi Seed Extract (Pangium edule Reinw.). Eugenia, 18(3): 186-197.

Salaki C. L., Paendong E., \& Pelealu J. (2012). Botanical Pesticides from Pangi (Pangium sp.) Leaf Extract Against Plutella xylostella Insects in North Sulawesi. Eugenia, 18 (3).

Salbiah, D. (2020). Test of Several Concentrations of Extract of Bintaro Leaf Powder (Cerbera manghas L.) Against Sweet Corn Cob Borer (Helicoverpa armigera Hubner). Dinamika Pertanian, 36 (1): 21-28.

Salbiah D., Rustam R., \& Daeli F. S. (2019). Test of Several Concentrations of Betel Nut Extract (Areca catechu L.) to Control 
Caterpillars (Chrysodeixis chalcites Esper) on Soybeans (Glycine max L.). Dinamika Pertanian, 35 (2): 51-58.

Samuel M., Oliver S. V., Coetzee M., \& Brooke B. D. (2016). The Larvicidal Effects of Black Pepper (Piper nigrum L.) and Piperine Against Insecticide Resistant and Susceptible Strains of Anopheles malaria Vector Mosquitoes. Parasites \& Vectors, 9 (1): 1-9.

Santhanam, G. \& Nagarajan S. (1990). Wound Healing Activity of Curcuma aromatica and Piper betle. Fitoterapia, 61:458-9.

Sarwar, M. (2015). The Dangers of Pesticides Associated with Public Health and Preventing of The Risks. International Journal of Bioinformatics and Biomedical Engineering, 1 (2): 130-136.

Scapinello J., de Oliveira J. V., Chiaradia L. A., Junior O. T., Niero R., \& Magro J. D. (2014). Insecticidal and Growth Inhibiting Action of the Supercritical Extracts of Melia azedarach on Spodoptera frugiperda. Storage and Processing of Agricultural Products, 18 (8): 866-872.

Scott I. M., Gagnon N., Lesage L., Philogene B. J. R., \& Arnason J. T. (2005). Efficacy of Botanical Insecticides from Piper Species (Piperaceae) Extracts for Control of European Chafer (Coleoptera: Scarabaeidae). Journal of Economic Entomology, 98 (3): 845-855.

Seiber J. N., Coats J., Duke S. O., \& Gross A. D. (2014). Botanical Pesticidess: State of The Art and Future Opportunities. Journal of Agricultural and Food Chemistry, 62 (48): 11613-11619.

Setiawan, A. N. \& Supriyadi A. (2014). Test the Effectiveness of Various Concentrations of Bintaro for Botanical Pesticide (Cerbera manghas) Against Armyworm (Spodoptera litura) on Soybeans. Planta Tropika: Jurnal Agrosains, 2 (2): 99-105.

Shafiei F., Ahmadi K., \& Asadi M. (2018). Evaluation of Systemic Effects of Four Plant Extracts Compared with Two Systemic Pesticides, Acetamiprid and Pirimicarb Through Leaf Spraying Against Brevicoryne brassicae L. (Hemiptera: Aphididae). Journal of Plant Protection Research, 58 (3): 258-264.

Shamim M. D., Khan N. A., \& Singh K. N. (2011). Inhibition of Midgut Protease of Yellow Stem Borer (Scirpophaga incertulas) by Cysteine Protease-Like
Inhibitor from Mature Jackfruit (Artocarpus heterophyllus) Seed. Acta Physiologiae Plantarum, 33 (6): 22492257.

Shaqi, A. M. (2021). The effect of course extract of jatropha curcas on the pest of the leaveroller wormer (Lamprosema indicata) (Lepidoptera, Pyralidae) on soybean (Glycine max) in the screen house. Unpublished undergraduate thesis in partial fulfillment of the requirements for the degree of Bachelor, North Sumatra State Islamic University, Medan, North Sumatera, Indonesian.

Sharon E. A., Velayutham K., \& Ramanibai R. (2018). Biosynthesis of Copper Nanoparticles Using Artocarpus heterophyllus Against Dengue Vector Aedes aegypti. Int J Life Sci Scienti Res, 2455 (1716): 1716.

Silva G. N., Faroni L. R. A., Sousa A. H., \& Freitas R. S. (2012). Bioactivity of Jatropha curcas L. to Insect Pests of Stored Products. Journal of Stored Products Research, 48: 111-113.

Simas N. K., Lima E. D. C., Kuster R. M., Lage C. L. S., \& Oliveira Filho A. M. D. (2007). Potential Use of Piper nigrum Ethanol Extract Against Pyrethroid-Resistant Aedes aegypti Larvae. Revista da Sociedade Brasileira de Medicina Tropical, 40 (4): 405-407.

Subaharan K., Senthoorraja R., Manjunath S., Thimmegowda G. G., Pragadheesh V. S., Bakthavatsalam N., Mohan M. G., SenthilNathan S., David K. J., Basavarajappa S., \& Ballal C. (2021). Toxicity, Behavioural and Biochemical Effect of Piper betle L. Essential Oil and Its Constituents Against Housefly, Musca domestica L. Pesticide Biochemistry and Physiology, 174 (2021): 104804.

Subekti, N. (2020). Toxicity Of Essential Oils Against Termite Macrotermes gilvus Hagen (Blattodea: Termitidae). In 6th International Conference on Mathematics, Science, and Education (ICMSE 2019), Oct. 9-10, Semarang, Indonesia.

Subrata, I. M. \& Rai I. G. A. (2019). Fungicidal Activity of Betel Leaves (Piper Betle L.) of beleng cultivar on Fusarium Oxysporum f. sp. Vanillae Causes Stem Rot in Vanilla. Emasains: Jurnal Edukasi Matematika dan Sains, 8 (1): 41-50. 
Subrata, I. M. (2016). Fungicide Activity of Betel Leaf (Piper betle L.) Cultivar Beleng Extract Against Fusarium solani var. Coeruleum Causes of Dry Root Disease in Potato Tuber (Solanum tuberosum L.). Emasains: Jurnal Edukasi Matematika dan Sains, 5 (2): 31-39.

Sukenti K., Hakim L., Indriyani S., Purwanto Y., \& Matthews P. J. (2016). Ethnobotanical Study on Local Cuisine of The Sasak Tribe in Lombok Island, Indonesia. Journal of Ethnic Foods, 3 (3): 189-200.

Sulistianingsih M., Jati A. N., \& Zahida F. (2014). Toxicity Test of Kluwak Seed Extract (Pangium edule Reinw.) as Molluscicide of Golden Snail (Pomacea caniculata Lamarck, 1804) on Rice. Jurnal Bahan Alam Terbarukan, 1 (2): 2407-2370.

Supiandi M. I., Mahanal S., Zubaidah S., Julung H., \& Ege B. (2019). Ethnobotany of Traditional Medicinal Plants Used by Dayak Desa Community in Sintang, West Kalimantan, Indonesia. Biodiversitas Journal of Biological Diversity, 20 (5): 1264-1270.

Susanti R., Risnawati, \& Fadhillah W. (2020). Primary Metabolite Qualitative Test of Bintaro Plant (Carbera odollam Gaertn) as a Pest Biopesticide Rattus Argentiventer. Jurnal Pertanian Tropik, 7 (3): 312-316.

Susilo A., Haryanta D., \& Sa'adah T. T. (2019). Response of Riptortus linearis Towards The Aplication of Bintaro (Cerbera manghas) Leaf Extract. EurAsian Journal of BioSciences, 13 (2): 2217-2224.

Suswando R., Djamilah D., \& Eko S. (2019). Effectiveness of areca (Areca catechu L.) seed extract in controlling Plutella xylostella L. in Pakcoy (Brassica rapa L.). Unpublished undergraduate thesis in partial fulfillment of the requirements for the degree of Bachelor, Bengkulu University, Bengkulu, Indonesia.

Sutriadi M. T., Harsanti E. S., Wahyuni S., \& Wihardjaka A. (2019). Botanical Pesticides: Prospects of Environmentally Friendly Pest Control. Jurnal Sumberdaya Lahan, 13(2): 89-101.

Tampil G. F., Salaki C. L., \& Memah V. (2020). Combination of Bitung Fruit (Barringtonia Asiatica L. Kurtz) and Pangi (Pangium Edule Reinw) Botanical Pesticides Against Vector Insects for Dengue Fever Disease Aedes aegypti. Sam
Ratulangi Journal of Entomology Review, 1 (1).

Tong L., Zhifang K., \& Xiaodong Z. (2007). Insecticidal Effect of Six Kinds of Solvent Extracts from Cerbera manghas on Anua indiscriminate. Journal of Northeast Forestry University, 35 (3): 59-63.

Tripathi A. K., Upadhyay S., Bhuiyan M., \& Bhattacharya P. R. (2009). A Review On Prospects of Essential Oils as Botanical Pesticides in Insect-Pest Management. Journal of Pharmacognosy and Phytotherapy, 1 (5): 052-063.

Turhadi T., Bedjo B., \& Suharjono S. (2020). The Effect of Bintaro (Cerbera odollam) leaf Extract on The Time to Stop Feeding And The Mortality of Gray Worn Services (Spodoptera litura). Agro Bali: Agricultural Journal, 3 (2): 136-143.

Tukimin S. W., Soetopo D., \& Karmawati E. (2010). Effect of Castor Oil (Jatropha curcas LINN.) on Mortality, Pupa Weight, and Egg Laying of Jatropha Pest. Jurnal Penelitian Tanaman Industri, 16 (4): 159164.

Upadhyay, R. K. (2013). Bio-efficacy of Latex Extracts from Plant Species Thevetia nerifolia, and Artocarpus heterophyllus, Ficus glomerata and Calotropis procera on Survival, Feeding, Development and Reproductive Behavior of Spodoptera litura (F.) Noctuidae: Lepidoptera. Int J Chem Biochem Sci, 4: 86-98.

Upadhyay, R. K \& Jaiswal, G. (2007). Evaluation of Biological Activities of Piper nigrum Oil Against Tribolium castaneum. Bulletin of Insectology, 60 (1): 57-61.

Vasantha-Srinivasan P., Chellappandian M., Senthil-Nathan S., Ponsankar A., Thanigaivel A., Karthi S., Edwin E. S., Selin-Rani S., Kalaivani K., Maggi F., \& Benelli G. (2018). A Novel Herbal Product Based on Piper betle and Sphaeranthus indicus Essential Oils: Toxicity, Repellent Activity and Impact on Detoxifying Enzymes GST and CYP450 of Aedes aegypti Liston (Diptera: Culicidae). Journal of Asia-Pacific Entomology, 21 (4): 1466-1472.

Vasantha-Srinivasan P., Senthil-Nathan S., Ponsankar A., Thanigaivel A., Edwin E. S., Selin-Rani S., Chellappandian M., Pradeepa V., Lija-Escaline J., Kalaivai K., Hunter W., Duraipandiyan V., \& Al-Dhabi N. A. (2017). Comparative Analysis of 
Mosquito (Diptera: Culicidae: Aedes aegypti Liston) Responses to The Insecticide Temephos and Plant Derived Essential Oil Derived from Piper betle L. Ecotoxicology and Environmental Safety, 139: 439-446.

Vasudevan K., Malarmagal R., Charulatha H., Saraswatula V. L., \& Prabakaran K. (2009). Larvicidal Effects of Crude Extracts of Dried Ripened Fruits of Piper nigrum Against Culex quinquefasciatus Larval Instars. Journal of Vector Borne Diseases, 46 (2): 153-156.

Verawati, N. (2018). Test The Effectiveness of Picung (Pangium edule) and Mahkota Dewa (Phaleria macrocarpa) Against Mortality of Earth Bomb (Scotinophora coarctata) on Pandanwangi Rice. Agroscience, 8 (2): 180-188.

Vite-Vallejo O., Barajas-Fernández M. G., Saavedra-Aguilar M., \& Cardoso-Taketa A. (2018). Insecticidal Effects of Ethanolic Extracts of Chenopodium ambrosioides, Piper nigrum, Thymus vulgaris, and Origanum vulgare against Bemisia tabaci1. Southwestern Entomologist, 43 (2): 383-393.

Wang, Z. (1984). Acute Rodenticides In The Control of Rodent Pest in China: a Review. In: Proceedings of the 11th Vertebrate Pest Conference, University of Nebraska, Lincoln, USA.

Warsa, J. H. (2018). Effectiveness testing of noni root extract (Morinda citrifolia L.) against mortality of leaf caterpillar (Plutella xylostella L.) on brocolli (Brassica oleracea L.) Unpublished undergraduate thesis in partial fulfillment of the requirements for the degree of Bachelor, Mataram University, Lombok, NTB, Indonesia.

Waroy, H.F. \& Utami S. (2020). The Food Plant Ethnobotany of Ampari Tribe Community in Papua, Indonesia. The 9th International Seminar on New Paradigm and Innovation of Natural Sciences and its Application, Oct. 22, Semarang, Indonesia.

Wiryadiputra S., Rusda I., \& Iis N. A. (2014). Effect of Picung (Pangium edule) Plant Extract as a Botanical Pesticide on Coffee Berry Borer Mortality. Pelita Perkebunan, 30 (3): 220-228.

Woyinka O. A, Oyewole I. O, Amos B. M. W., \& Onasoga O. F. (2006). Comparative Pesticidal Activity of Dichloromethane
Extracts of Piper nigrum Against Sitophilus zeamais and Callosobruchus maculatus. African Journal of Biotechnology, 5 (24): 2446-2449.

Yoon M. Y., Cha B., \& Kim J. C. (2013). Recent Trends in Studies on botanical Fungicides in Agriculture. The Plant Pathology Journal, 29 (1): 1-9.

Yulianti, R. (2021). The effectiveness of kepayang fruit extract (Pangium edule Reinw) on termite food rate and mortality coptotermes gestroi. Unpublished undergraduate thesis in partial fulfillment of the requirements for the degree of Bachelor, Raden Intan State Islamic University, Lampung, Indonesian.

Yuningsih, Y. \& Kartina G. (2007). Effectiveness of Picung (Pangium edule Reinw.) Seed Extract on The Mortality of The Golden Snail (Pomacea canaliculate Lamck.). Berita Biologi, 8 (4): 307-310.

Wati I. F., Efri E., \& Maryono T. (2014). The Effectiveness of Betel Leaf Extract and Babadotan Leaf in Controlling Anthracnose Disease in Chili Fruit (Capsicum annum L.). Jurnal Agrotek Tropika, 2 (3): 436-440.

Werrie P.Y., Durenne B., Delaplace P., \& Fauconnier M. L. (2020). Phytotoxicity of Essential Oils: Opportunities and Constraints for The Development of Botanical Pesticidess. Foods, 9 (9): 1-24.

Zailani, H. F. (2015). Test the effectiveness of botanical rodenticides from bintaro fruit extract (Cerbera manghas Boiteau, Pierre L.) against rat pests. Unpublished undergraduate thesis in partial fulfillment of the requirements for the degree of Bachelor, Jember University, Jember, East Java, Indonesia. 